\title{
Méthodes à $N$ Corps pour un Problème de Milieux Pluristratifiés Perturbés
}

\author{
par \\ Yves DeRmenJian* et Viorel IfTIMIE**
}

\begin{abstract}
On étudie l'opérateur $H:=\nabla^{*} \rho \nabla+V$ dans $L^{2}(X), X$ espace euclidien réel de dimension finie, où $V$ est un potentiel du type " $N$ corps" associé à une famille finie $\mathscr{L}$ de sous-espaces vectoriels de $X$ et $\rho$ admet une décomposition suivant $\mathscr{L}$, compatible avec celle de $V$. Chaque composante de $V$, respectivement $\rho$, est une somme de perturbations de type "courte portée" et "longue portée". En utilisant une variante de la méthode de Mourre [14] ainsi que des idées de la théorie du problème à $N$ corps de la mécanique quantique, on fait l'analyse spectrale de l'opérateur $H$ et on prouve un principe d'absorption limite.
\end{abstract}

\section{§1. Introduction}

Soit $X$ un espace euclidien réel de dimension finie dont on désigne le produit scalaire (étendu en tant que fonctionnelle bilinéaire au complexifié) de deux éléments $x$ et $y$ par $x . y$. Si $x \in X \otimes \mathbb{C}$, on pose $|x|^{2}:=x . \bar{x}$; $d x$ sera la mesure riemanienne sur $X$. On note par $\nabla$ l'opérateur "gradient" et par $\nabla^{*}$ l'opérateur "divergence" (l'adjoint formel de $\nabla$ ). Alors $\Delta:=\nabla^{*} \nabla$ sera l'opérateur de Laplace-Beltrami sur $X$ (lorsque $X=\mathbb{R}^{n}$, on aura $\Delta=-\sum_{1 \leq j \leq n} \partial_{j}^{2}$ ).

On considère une famille finie $\mathscr{L}$ de sous-espaces vectoriels de $X$, qui vérifie I-(i) $O \in \mathscr{L}, X \in \mathscr{L}$.

(ii) Pour tous $Y, Z \in \mathscr{L}$, la somme vectorielle $Y+Z \in \mathscr{L}$.

On pose $\mathscr{L}_{1}:=\mathscr{L} \backslash\{X\}$ et si $Y \in \mathscr{L}, \mathscr{L}(Y):=\{Z \in \mathscr{L} ; Z \subset Y\}$, tandis que $Y^{\perp}$ désigne le complémentaire orthogonal de $Y$ dans $X: Y^{\perp}:=X \ominus Y$.

On définit de façon standard les espaces de Sobolev usuels $\mathscr{H}^{s}(X), s \in \mathbb{R}$. En particulier, $\mathscr{H}^{0}(X)=L^{2}(X)$.

Communiqué par T. Kawai, le 18 février 1999.

1991 Mathematics Subject Classification(s): 35P25, 47A55, 81F10

* LAPT, UMR-CNRS $\mathrm{n}^{\circ}$ 6632, CMI, Université de Provence, 39, rue Joliot Curie, 13453 Marseille Cedex 13, France.

** Université de Bucarest, 14 rue Academiei, Bucarest, Roumanie. 
Si $E$ et $F$ sont deux espaces de Banach, on désigne par $\mathscr{B}(E, F)$ (resp. $\mathscr{K}(E, F)$ ) l'ensemble des opérateurs linéaires et bornés (resp. compacts) de $E$ dans $F$. En particulier, $\mathscr{B}(E):=\mathscr{B}(E, E), \mathscr{K}(E):=\mathscr{K}(E, E)$.

Pour tout $Y \in \mathscr{L}$ on se donne les fonctions réelles $\delta^{Y}$ et $V^{Y}$ définies sur $Y$, telles que $\delta^{0}=0, V^{0} \in \mathbb{R}$ et pour $Y \neq 0, \delta^{Y}=\delta^{Y, S}+\delta^{Y, L}, V^{Y}=V^{Y, S}+V^{Y, L}$. On se donne aussi deux constantes $\rho^{0} \in \mathbb{R}_{+}^{*}$, et $\theta \in(0,1]$ telles que les hypothèses suivantes soient vérifiées:

II-( i ) $\delta^{Y, S}$ est une perturbation du type "courte portée", à savoir $\langle.\rangle^{1+\theta} \delta^{Y, S} \in$ $L^{\infty}(Y)$, où $\langle x\rangle:=\left(1+|x|^{2}\right)^{1 / 2}, x \in X$.

(ii) $\delta^{Y, L}$ est une perturbation du type "longue portée", c'est-à-dire $\langle.\rangle^{\theta} \delta^{Y, L}$ $\in L^{\infty}(Y),\langle.\rangle^{1+\theta} \nabla \delta^{Y, L} \in L^{\infty}(Y, Y)$.

(iii) Pour tout $Y \in \mathscr{L}, \rho^{Y}:=\rho^{0}+\sum_{z \in \mathscr{L}(Y)} \delta^{Z} \otimes 1_{Y \ominus Z}>0$ et $1 / \rho^{Y} \in L^{\infty}(Y)$. III-( i ) $V^{Y, S}, \quad V^{Y, L} \in L_{\ell o c}^{1}(Y)$ et l'opérateur de multiplication $V^{Y} \in$ $\mathscr{K}\left(\mathscr{H}^{1}(Y), \mathscr{H}^{-1}(Y)\right)$.

(ii) $V^{Y, S}$ est une perturbation du type "courte portée", c'est-à-dire $\langle.\rangle^{1+\theta} V^{Y, S} \in \mathscr{B}\left(\mathscr{H}^{1}(Y), \mathscr{H}^{-1}(Y)\right)$.

(iii) $V^{Y, L}$ est une perturbation du type "longue portée", ce qui signifie que $\langle.\rangle^{\theta} V^{Y, L} \in \mathscr{B}\left(\mathscr{H}^{1}(Y), \mathscr{H}^{-1}(Y)\right)$, et pour tout $\xi \in Y,\langle.\rangle^{1+\theta} \xi . \nabla V^{Y, L} \in$ $\mathscr{B}\left(\mathscr{H}^{1}(Y), \mathscr{H}^{-1}(Y)\right)$, uniformément pour $|\xi| \leq 1$.

On introduit les fonctions suivantes, définies sur $X$ :

$$
\begin{gathered}
\delta_{Y}=\delta^{Y} \otimes 1_{Y^{\perp}}, \quad \delta_{Y}^{S}:=\delta^{Y, S} \otimes 1_{Y^{\perp}}, \quad \delta_{Y}^{L}:=\delta^{Y, L} \otimes 1_{Y^{\perp}}, \quad \rho_{Y}:=\rho^{Y} \otimes 1_{Y^{\perp}}, \\
V_{Y}:=V^{Y} \otimes 1_{Y^{\perp}}, \quad V_{Y}^{S}:=V^{Y, S} \otimes 1_{Y^{\perp}}, \quad V_{Y}^{L}:=V^{Y, L} \otimes 1_{Y^{\perp}}
\end{gathered}
$$

et finalement $\rho:=\rho_{X}, V=\sum_{Y \in \mathscr{L}} V_{Y}$.

Si $\mathscr{H}$ est un espace de Hilbert, on désigne par $(., .)_{\mathscr{H}}$ son produit scalaire et par $\|.\|_{\mathscr{H}}$ la norme associée. Si $\mathscr{H}=L^{2}(X)$ on écrit tout simplement $(.,$.$) pour$ $(.,)_{L^{2}(X)}$ et $\|\cdot\|$ pour $\|\cdot\|_{L^{2}(X)}$.

Considérons la forme quadratique symétrique $h(\rho, V)$ sur $\mathscr{H}=L^{2}(X)$, de domaine $\mathscr{H}^{1}(X)$ et définie par

$$
h(\rho, V)(u, v):=\int_{X} \rho \nabla u \cdot \nabla \bar{v} d x+(V u, v), \quad u, v \in \mathscr{H}^{1}(X),
$$

où l'on désigne aussi par $(.,$.$) l'extension du produit scalaire de L^{2}(X)$ en tant que fonctionnelle sesquilinéaire sur $\mathscr{H}^{-1}(X) \times \mathscr{H}^{1}(X)$ ou bien sur $\mathscr{D}^{\prime}(X) \times$ $C_{0}^{\infty}(X)$.

La forme $h(\rho, 0)$ est fermée, $C_{0}^{\infty}(X)$ en est un domaine essentiel et il existe une constante $c>0$ telle que $h(\rho, 0)(u, u) \geq c\||\nabla u|\|^{2}, u \in \mathscr{H}^{1}(X)$. D'autre part, d'après l'hypothèse III-(i), la forme $h(0, V)$ est relativement bornée par rapport à $h(\rho, 0)$, de borne relative égale à zéro. Alors $h(\rho, V)$ est symétrique, fermée, inférieurement semi-bornée, $C_{0}^{\infty}(X)$ en étant un domaine essentiel. Il 
existe donc un unique opérateur $H=H(\rho, V)$ auto-adjoint sur $\mathscr{H}$, semi-borné inférieurement et qui vérifie

$$
h(\rho, V)(u, v)=(H u, v), \quad u \in D(H), \quad v \in \mathscr{H}^{1}(X) .
$$

On peut considérer $H \in \mathscr{B}\left(\mathscr{H}^{1}(X), \mathscr{H}^{-1}(X)\right)$ et alors

$$
H u=\nabla^{*} \rho \nabla u+V u, \quad u \in \mathscr{H}^{1}(X) .
$$

Dans ce cadre, on remarque que $(H-\lambda)^{-1} \in \mathscr{B}\left(\mathscr{H}^{-1}(X), \mathscr{H}^{1}(X)\right)$ si $\lambda \in$ $\mathbb{C} \backslash \sigma(H)$.

Remarque 1.1. $H(1, V)$ a la forme du hamiltonien d'un système à $N$ corps dans le formalisme de Agmon-Froese-Herbst (voir [12], [1]), tandis que $H(\rho, 0)$ pourrait être considéré comme le propagateur associé à un milieu pluristratifié. En particulier, le cas d'un milieu simplement stratifié (voir [10], [6]) est obtenu pour $X=\mathbb{R}^{m+n}$ et $\mathscr{L}=\left\{0, \mathbb{R}^{m} \times\{0\}, \mathbb{R}^{m+n}\right\}$.

Le premier résultat est un théorème du type $H V Z$, permettant de calculer le spectre essentiel de $H$ en fonction des spectres des opérateurs

$$
H_{Y}:=H\left(\rho_{Y}, V-I_{Y}\right), \quad Y \in \mathscr{L}_{1}, \quad \text { où } I_{Y}:=\sum_{Z \in \mathscr{L} \backslash \mathscr{L}(Y)} V_{Z} .
$$

En général, si $H$ est un opérateur auto-adjoint dans un espace de Hilbert $\mathscr{H}$, on désigne par $\sigma(H)$ (resp. $\sigma_{\text {ess }}(H), \sigma_{\text {cont }}(H), \sigma_{s c}(H), \sigma_{a c}(H), \sigma_{p}(H)$ ) le spectre de $H$ (resp. le spectre essentiel, continu, continu singulier, absolument continu, l'ensemble des valeurs propres).

Théorème 1.2. Sous les hypothèses I, II et III-(i) on a

(a) $\sigma_{\text {ess }}(H)=\bigcup_{Y \in \mathscr{L}_{1}} \sigma_{\text {ess }}\left(H_{Y}\right)$.

(b) Pour tout $Y \in \mathscr{L}_{1}, \sigma_{p}\left(H_{Y}\right)=\varnothing$, donc $\sigma_{\text {ess }}\left(H_{Y}\right)=\sigma\left(H_{Y}\right)=\sigma_{\text {cont }}\left(H_{Y}\right)$.

(c) En particulier, $\sigma(H(\rho, 0))=\sigma_{\text {ess }}(H(\rho, 0))=[0, \infty[$.

Pour obtenir des propriétés spectrales plus profondes de $H, y$ compris un principe d'absorption limite, on appliquera la méthode de Mourre [14], telle qu'elle a été développée dans [15], [12], [5], [2] pour des hamiltoniens à $N$ corps, en utilisant à fond la partition de l'unité de Ruelle-Simon [9] pour faire des démonstrations par récurrence. Usuellement, l'opérateur conjugué est le générateur des dilatations $A:=\frac{1}{2}(x . D+D . x), D:=-i \nabla$, qui a la propriété de décomposition très remarquable suivante: pour tout $Y \in \mathscr{L}, A=A_{Y} \otimes 1_{Y^{\perp}}+$ $1_{Y} \otimes A_{Y^{\perp}}$, où $A_{Y}$ est le générateur des dilatations sur $Y$. Ce choix ne convient pas pour l'étude de $H(\rho, V)$ si l'on veut travailler avec des perturbations $\delta^{Y}$ assez singulières. Ainsi, on est amené à chercher l'opérateur conjugué $A$ sous la forme d'un pseudo-différentiel presque-local dépendant d'un paramètre déterminé en fonction de l'intervalle spectral (idée qui n'est pas tout à fait 
nouvelle: voir [6]). Afin de construire $A$ tel qu'il admette une décomposition comme ci-dessus relativement à $\mathscr{L}$, il faut que cette famille vérifie une hypothèse supplémentaire.

Un élément $Y \in \mathscr{L} \backslash\{0\}$ est appelé minimal si pour tout $Z \in \mathscr{L} \backslash\{0\}$ tel que $Z \subset Y$, on a $Z=Y$. On désigne par $\mathscr{L}_{m}$ l'ensemble des éléments minimaux de $\mathscr{L}$. On peut supposer (en augmentant éventuellement $\mathscr{L}$ ) que $\mathscr{L}_{m}$ engendre $\mathscr{L}$, c'est-à-dire que tout élément de $\mathscr{L}$ est une somme vectorielle finie d'éléments de $\mathscr{L}_{m}$. On fait l'hypothèse suivante:

IV - Les éléments de $\mathscr{L}_{m}$ sont mutuellement orthogonaux.

On en déduit que tout $Y \in \mathscr{L} \backslash\{0\}$ est la somme directe orthogonale des éléments de $\mathscr{L}_{m}(Y):=\mathscr{L}(Y) \cap \mathscr{L}_{m}$. De plus, si $Y \in \mathscr{L}$, alors $Y^{\perp}$ appartient à $\mathscr{L}$.

Avant de formuler les résultats principaux de ce papier, remarquons que pour tout $Y \in \mathscr{L}$, on peut construire un opérateur $H^{Y}$ auto-adjoint sur $L^{2}(Y)$, à partir des familles $\mathscr{L}(Y),\left\{\delta^{Z}\right\}_{Z \in \mathscr{L}(Y)}$ et $\left\{V^{Z}\right\}_{Z \in \mathscr{L}(Y)}$. Par convention $L^{2}(0)=$ $\mathbb{C}$ et $H^{0}=V^{0}$.

Théorème 1.3. Sous les hypothèses I à IV on a:

(a) L'ensemble des seuils $\tau(H):=\bigcup_{Y \in \mathscr{L}_{1}} \sigma_{p}\left(H^{Y}\right)$ est dénombrable et fermé.

(b) Les éléments de $\sigma_{p}(H) \backslash \tau(H)$ sont des valeurs propres de multiplicité finie, qui ne peuvent s'accumuler qu'aux points de $\tau(H)$. En particulier, $\tau(H) \cup$ $\sigma_{p}(H)$ est un ensemble dénombrable et fermé.

(c) $\sigma_{s c}(H)=\phi$.

Pour formuler le principe d'absorption limite nous utilisons certains espaces de Sobolev à poids (voir [4], [5]). Soient $\alpha, \beta \in C_{0}^{\infty}(X)$ réelles telles que $\alpha(x)>0$ si $1 / 2<|x|<2, \alpha(x)=0$ si $|x| \leq 1 / 2$ ou bien si $|x| \geq 2, \beta(x)>0$ si $|x|<2, \beta(x)=0$ si $|x| \geq 2$. Pour $s, t \in \mathbb{R}$ et $1 \leq q \leq \infty$ nous désignerons par $\mathscr{H}_{t, q}^{s}(X)$ l'espace de Banach des distributions $u \in \mathscr{S}^{\prime}(X)$ telles que

$$
\|u\|_{\mathscr{H}_{t, q}^{s}(X)}:=\left\|\langle D\rangle^{s} \beta u\right\|+\left[\int_{1}^{\infty}\left\|\langle D\rangle^{s} r^{t} \alpha(. / r) u\right\|^{q} r^{-1} d r\right]^{1 / q}<\infty .
$$

Pour $q=\infty$ on convient que le second terme du côté droit doit être remplacé par sup $\left\|\langle D\rangle^{s} r^{t} \alpha(. / r) u\right\|$. L'opérateur pseudo-différentiel $\langle D\rangle^{s}$ a pour symbole $\langle.\rangle^{s}$.

On note aussi $\mathscr{H}_{t}^{s}(X):=\mathscr{H}_{t, 2}^{s}(X)$; c'est l'espace de Sobolev à poids usuel défini par la norme $\left\|\langle D\rangle^{s}\langle.\rangle^{t} u\right\|$. En particulier, $\mathscr{H}^{s}(X)=\mathscr{H}_{0}^{s}(X)$.

Soit $\mathbb{C}^{ \pm}:=\{\lambda \in \mathbb{C} ; \pm \Im ⿱ 一 ⿻ 上 丨 m \lambda \lambda>0\}$.

Théorème 1.4. Sous les hypothèses I à IV, on a:

(a) Les applications

$$
\mathbb{C}^{ \pm} \ni \lambda \mapsto(H-\lambda)^{-1} \in \mathscr{B}\left(\mathscr{H}_{(1 / 2), 1}^{-1}(X), \mathscr{H}_{-(1 / 2), \propto}^{1}(X)\right)
$$


sont bien définies, holomorphes et admettent des extensions continues pour la topologie faible ̀̀ $\mathbb{C}^{ \pm} \cup\left(\mathbb{R} \backslash\left[\tau(H) \cup \sigma_{p}(H)\right]\right)$.

(b) Les applications

$$
\mathbb{C}^{ \pm} \ni \lambda \mapsto(H-\lambda)^{-1} \in \mathscr{B}\left(\mathscr{H}_{\gamma^{\prime}}^{-1}(X), \mathscr{H}_{-\gamma}^{1}(X)\right)
$$

sont bien définies pour tout $\gamma>1 / 2$, holomorphes et admettent des extensions continues pour la topologie uniforme à $\mathbb{C}^{ \pm} \cup\left(\mathbb{R} \backslash\left[\tau(H) \cup \sigma_{p}(H)\right]\right)$.

Remarque 1.5. Les théorèmes 1.3 et 1.4 restent valables sans l'hypothèse IV. Il suffit que dans l'hypothèse II on suppose $\delta^{Y, S}=0$ pour tout $Y \in \mathscr{L}$ et, qu'à la place de l'hypothèse III, on ait

III'-(i) Pour tout $Y \in \mathscr{L}, V^{Y} \in L_{\ell o c}^{2}(X ; \mathbb{R})$ et l'opérateur de multiplication avec $V^{Y}$ appartient à $\mathscr{K}\left(\mathscr{H}^{2}(Y), L^{2}(Y)\right)$.

(ii) $\mathrm{Si} A^{Y}$ est le générateur des dilatations sur $Y$, alors $\langle.\rangle^{\theta} V^{Y}$ et $\langle.\rangle^{\theta}\left[V^{Y}, A^{Y}\right]$ appartient à $\mathscr{B}\left(\mathscr{H}^{2}(Y), \mathscr{H}^{-1}(Y)\right)$.

Par exemple, une fonction $V^{Y}$ qui vérifie (i) et de plus $V^{Y}=V^{Y, S}+V^{Y, L}$, où $V^{Y, S}, V^{Y, L} \in L_{\ell o c}^{2}(X ; \mathbb{R})$ et

(a) $\langle.\rangle^{1+\theta} V^{Y, S} \in \mathscr{B}\left(\mathscr{H}^{2}(Y), L^{2}(Y)\right)$,

(b) $\langle.\rangle^{\theta} V^{Y, L} \in \mathscr{B}\left(\mathscr{H}^{2}(Y), L^{2}(Y)\right),\langle y\rangle^{\theta} y \cdot \nabla V^{Y, L} \in \mathscr{B}\left(\mathscr{H}^{2}(Y), \mathscr{H}^{-1}(Y)\right)$, vérifie aussi (ii).

Il est évident que sous les hypothèses de cette remarque, $D(H)=\mathscr{H}^{2}(X)$.

Remarque 1.6. Le but de ce papier est en fait l'étude spectrale de l'opérateur $H(\rho, 0)$, régissant la propagation des ondes dans des milieux pluristratifiés (d'où l'intérêt de considérer des fonctions $\rho$ assez singulières). $\mathrm{Au}$ cours de la démonstration de l'inégalité de Mourre il s'est avéré que le bon opérateur à étudier du point de vue mathématique est $H(\rho, V)$, d'où l'idée de faire une théorie unifiée pour les milieux pluristratifiés et les systèmes à " $N$ corps".

Remarque 1.7. Les hypothèses II et III ne sont pas les plus générales possibles, surtout en ce qui concerne les conditions de décroissance à l'infini. On pourrait aussi considérer des perturbations $V^{Y}$ qui ne soient pas des opérateurs locaux.

Remarque 1.8. Les opérateurs $H_{Y}, Y \in \mathscr{L}_{1}$, possèdent une propriété de décomposition importante. Plus exactement, on a au sens des formes

$$
H_{Y}=H^{Y} \otimes 1_{Y^{\perp}}+\rho^{Y} \otimes \Delta_{Y^{\perp}},
$$

où $\Delta_{Y^{\perp}}$ est l'opérateur de Laplace-Beltrami sur $Y^{\perp}$. Cette relation permet d'obtenir directement des renseignements spectraux importants sur $H_{Y}$ si $\rho=1$; si $\rho$ n'est pas une constante, ceci est beaucoup plus difficile. 
De plus, $H(1, V)$ est déduit de $\Delta$ par une perturbation infiniment petite, ce qui est faux pour $H(\rho, V)$. Il est donc naturel que certaines idées de la théorie à $N$ corps s'appliquent au cas où $\rho$ n'est pas constant, bien que la technique soit assez différente.

Remarque 1.9. Les opérateurs $H(\rho, 0)$ ont été étudiés complètement pour les milieux simplement stratifiés (voir [10] et [6] pour la méthode de l'opérateur conjugué). Par contre, il n'y a que peu de résultats pour des opérateurs qui, dans un certain sens, correspondent aux milieux bistratifiés (voir [8] pour une méthode basée sur les fonctions propres généralisées et [11] pour la méthode de l'opérateur conjugué).

Le contenu du papier est le suivant: dans la seconde section on rappelle (en utilisant [5], [4], [2]) les résultats nécessaires de la méthode de l'opérateur conjugué. La section 3 est consacrée à certaines propriétés générales de l'opérateur $H(\rho, V)$, ainsi qu'à la démonstration des propriétés (a) et (c) du théorème 1.2. Dans la quatrième section on introduit l'opérateur conjugué et on prouve certaines propriétés auxiliaires. Dans la section 5 on étudie la régularité de $H(a, V)$ par rapport à l'opérateur conjugué et on prouve la propriété (b) du théorème 1.2. Finalement, dans la dernière section on prouve l'inégalité de Mourre et les théorèmes 1.3 et 1.4 .

On utilise les notations classiques pour certains espaces de fonctions et de distributions. On désigne par le même symbole une fonction et l'opérateur de multiplication par la même fonction.

\section{§2. La Méthode de l'Opérateur Conjugué}

Soient $\mathscr{G}$ et $\mathscr{H}$ deux espaces de Hilbert complexes et séparables, tels que $\mathscr{G} \subset \mathscr{H}$ continûment et densément (on dit que $(\mathscr{G}, \mathscr{H})$ est un couple de Friedrichs). Si l'on identifie $\mathscr{H}$ avec son adjoint, on aura $\mathscr{G} \subset \mathscr{H} \subset \mathscr{G}^{*}$ et on pourra aussi prolonger le produit scalaire $(.,$.$) de \mathscr{H}$ à une forme sesquilinéaire sur $\mathscr{G}^{*} \times \mathscr{G}$, ou bien sur $\mathscr{G} \times \mathscr{G}^{*}$.

On considère aussi un groupe unitaire dans ce couple, c'est-à-dire un $C_{0^{-}}$ groupe $\left\{W_{\tau}\right\}_{\tau \in \mathbb{R}}$ dans $\mathscr{G}^{*}$, qui laisse invariant les espaces $\mathscr{H}$ et $\mathscr{G}$ et qui est unitaire sur $\mathscr{H}$. Soit $A$ le générateur de ce groupe, défini sur $D\left(A ; \mathscr{G}^{*}\right)$.

On note $D(A ; \mathscr{H}):=\left\{u \in \mathscr{H} \cap D\left(A ; \mathscr{G}^{*}\right) ; A u \in \mathscr{H}\right\}$ et $D(A ; \mathscr{G}):=\{u \in \mathscr{G} \cap$ $\left.D\left(A ; \mathscr{G}^{*}\right) ; A u \in \mathscr{G}\right\}$. La restriction de $A$ à $\mathscr{H}$ est un opérateur auto-adjoint sur $\mathscr{H}$ de domaine $D(A ; \mathscr{H})$ et $W_{\tau}=e^{i \tau A}$ sur $\mathscr{H}$ pour tout $\tau \in \mathbb{R}$. De plus, la restriction de $A$ à $\mathscr{G}$ sera le générateur de la restriction du groupe $\left\{W_{\tau}\right\}_{\tau \in \mathbb{R}}$ à $\mathscr{G}$, de domaine $D(A ; \mathscr{G})$.

Définition 2.1. Soient $H \in \mathscr{B}\left(\mathscr{G}, \mathscr{G}^{*}\right)$ et $\theta \in(0,1]$. On dit que $H \in$ $C^{\theta}\left(A ; \mathscr{G}, \mathscr{G}^{*}\right)$ s'il existe une constante $C>0$ telle que 


$$
\left\|\left[W_{\tau}, H\right]\right\|_{\mathscr{B}\left(\mathscr{G}, \mathscr{G}^{*}\right)} \leq C|\tau|^{\theta} \text { si } \tau \in[-1,1],
$$

où le commutateur $\left[W_{\tau}, H\right]:=W_{\tau} H-H W_{\tau}$ est bien défini sur $\mathscr{G}$.

On note $C^{\theta}(A ; \mathscr{H}):=C^{\theta}(A ; \mathscr{H}, \mathscr{H})$.

Remarque 2.2. (Voir lemme 2.4 de [5]). Un opérateur $H \in \mathscr{B}\left(\mathscr{G}, \mathscr{G}^{*}\right)$ est de classe $C^{1}\left(A ; \mathscr{G}, \mathscr{G}^{*}\right)$ si et seulement si le commutateur $[H, A]$ défini en tant que forme sesquilinéaire sur $D(A ; \mathscr{G})$ par

$$
([H, A] u, v):=(H A u, v)-(H u, A v), u, v \in D(A ; \mathscr{G}),
$$

est continu pour la topologie induite par $\mathscr{G}$. Dans cette situation, on pourra identifier cette forme avec un opérateur de $\mathscr{B}\left(\mathscr{G}, \mathscr{G}^{*}\right)$.

Remarque 2.3. Si $H \in \mathscr{B}\left(\mathscr{G}, \mathscr{G}^{*}\right)$ est symétrique (c'est-à-dire $(H u, v)=$ $(u, H v)$ pour tous $u, v \in \mathscr{G})$ alors $(H u, u) \in \mathbb{R}$ pour tout $u \in \mathscr{G}$. De plus, si $H \in$ $C^{1}\left(A ; \mathscr{G}, \mathscr{G}^{*}\right)$, alors $i[H, A] \in \mathscr{B}\left(\mathscr{G}, \mathscr{G}^{*}\right)$ est lui aussi symétrique.

Théorème 2.4. (du viriel: voir le corollaire 2.5 de [5]). Si $H \in \mathscr{B}\left(\mathscr{G}, \mathscr{G}^{*}\right)$ est symétrique, de classe $C^{1}\left(A ; \mathscr{G}_{,} \mathscr{G}^{*}\right)$ et si $u, v \in \mathscr{G}, \lambda \in \mathbb{R}$, vérifient $H u=\lambda u$, $H v=\lambda v$, alors

$$
([H, A] u, v)=0
$$

Définition 2.5. Soient $H \in \mathscr{B}\left(\mathscr{G}, \mathscr{G}^{*}\right)$ et $\theta \in(0,1]$. On dit que $H \in$ $C^{1+\theta}\left(A ; \mathscr{G}, \mathscr{G}^{*}\right)$ si $H \in C^{1}\left(A ; \mathscr{G}, \mathscr{G}^{*}\right)$ et $[H, A] \in C^{\theta}\left(A ; \mathscr{G}, \mathscr{G}^{*}\right)$.

On note $C^{1+\theta}(A ; \mathscr{H}):=C^{1+\theta}(A ; \mathscr{H}, \mathscr{H})$.

Supposons dès maintenant que $H$ est un opérateur auto-adjoint sur $\mathscr{H}$ et que $\mathscr{G}$ est le domaine de sa forme (c'est-à-dire $\mathscr{G}=D\left(|H|^{1 / 2}\right.$ ). Alors $H \in$ $\mathscr{B}\left(\mathscr{G}, \mathscr{G}^{*}\right)$ et pour toute fonction $f: \mathbb{R} \rightarrow \mathbb{R}$, borélienne et bornée, $f(H)$ est un opérateur auto-adjoint borné sur $\mathscr{H}$ et, de plus, $f(H) \in \mathscr{B}(\mathscr{G}) \cap \mathscr{B}\left(\mathscr{G}^{*}\right)$.

Définition 2.6. Si $H \in C^{1}\left(A ; \mathscr{G}, \mathscr{G}^{*}\right)$ et $\lambda \in \mathbb{R}$, on dit que $A$ est conjugué à $H$ en $\lambda$ s'il existe $a>0, f \in C_{0}^{\infty}(\mathbb{R})$ réelle, $f(\lambda) \neq 0$ et $K \in \mathscr{K}\left(\mathscr{G}, \mathscr{G}^{*}\right)$ symétrique tels que l'on vérifie l'inégalité de Mourre

$$
f(H) i[H, A] f(H) \geq a f(H)^{2}+K,
$$

au sens des opérateurs de $\mathscr{B}\left(\mathscr{G}, \mathscr{G}^{*}\right)$.

Remarque 2.7. L'ensemble $\mu^{A}(H):=\{\lambda \in \mathbb{R} ; A$ est conjugué à $H$ en $\lambda\}$ est ouvert. En utilisant le théorème 2.4 , on voit que $\mu^{A}(H) \cap \sigma_{p}(H)$ est un sous-ensemble discret de $\mu^{A}(H)$ dont les éléments sont des valeurs propres de multiplicité finie de $H$.

On note $\mathscr{E}:=\left(\mathscr{G}^{*}, D\left(A ; \mathscr{G}^{*}\right)\right)_{(1 / 2), 1}$, l'espace de Banach obtenu par interpolation réelle ([3], [2]). On a $D\left(A, \mathscr{G}^{*}\right) \subset \mathscr{E} \subset \mathscr{G}^{*}$ continûment et densément, 
donc $\mathscr{G} \subset \mathscr{E}^{*}$ continûment (mais pas forcément densément). Par conséquent on a un plongement continu $\mathscr{B}\left(\mathscr{G}^{*}, \mathscr{G}\right) \subset \mathscr{B}\left(\mathscr{E}, \mathscr{E}^{*}\right)$. On peut donc considérer les fonctions holomorphes $\mathbb{C}^{ \pm} \ni \lambda \mapsto(H-\lambda)^{-1} \in \mathscr{B}\left(\mathscr{E}, \mathscr{E}^{*}\right)$.

Théorème 2.8. (voir le théorème 3.1 de [5]). Supposons que $H$ est un opérateur auto-adjoint sur $\mathscr{H}$, de classe $C^{1+\theta}\left(A ; \mathscr{G}, \mathscr{G}^{*}\right)$, où $\mathscr{G}=D\left(|H|^{1 / 2}\right)$ et $\theta \in$ $(0,1]$. Alors les fonctions holomorphes $\mathbb{C}^{ \pm} \ni \lambda \mapsto(H-\lambda)^{-1} \in \mathscr{B}\left(\mathscr{E}, \mathscr{E}^{*}\right)$ admettent des extensions continues pour la topologie faible à $\mathbb{C}^{ \pm} \cup\left[\mu^{A}(H) \backslash \sigma_{p}(H)\right]$.

En particulier, $\sigma_{s c}(H) \cap \mu^{A}(H)=\varnothing$.

\section{§3. Propriétés Générales}

Dans cette section on considère l'opérateur $H=H(\rho, V)$ défini par (1.2) et (1.3), sous les hypothèses I, II et III-(i) et on établit certaines propriétés qui le relie aux opérateurs $H_{Y}, Y \in \mathscr{L}_{1}$.

Lemme 3.1. Soient $\varphi \in C^{1}(X)$ telle que $\nabla \varphi$ soit borné et $\lambda \in \mathbb{C} \backslash \sigma(H)$. Il existe alors un opérateur $\left.T=T(\varphi, \lambda) \in \mathscr{B}^{\left(\mathscr{H}^{-1}\right.}(X), \mathscr{H}^{1}(X)\right)$ qui vérifie

$$
\varphi(H-\lambda)^{-1} f=(H-\lambda)^{-1}(\varphi f)+T f
$$

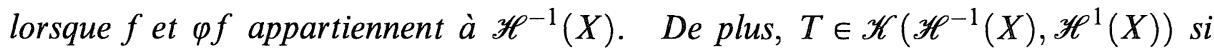
$\lim _{|x| \rightarrow \infty}|\nabla \varphi(x)|=0$.

Démonstration. A partir de la forme quadratique définissant $H$, en le considérant comme opérateur de $\mathscr{B}\left(\mathscr{H}^{1}(X), \mathscr{H}^{-1}(X)\right)$, on prouve que pour tout $u \in \mathscr{H}^{1}(X)$ et tout $\psi \in C_{0}^{1}(X)$,

$$
H(\psi u)=\psi H u-\rho \nabla \psi \cdot \nabla u+\nabla^{*}(\rho u \nabla \psi) .
$$

On choisit $\psi \in C_{0}^{\infty}(X)$ telle que $\psi(x)=1$ si $|x| \leq 1$ et pour $k \geq 1$ on pose $\psi_{k}(x):=\psi(x / k), x \in X$. Si $f$ a les propriétés de l'énoncé et $u:=(H-\lambda)^{-1} f$, on a, d'après (3.2), $(H-\lambda)\left(\psi_{k} \varphi u\right)=g_{k}, \quad$ où $\quad g_{k}:=\psi_{k} \varphi f-\rho \nabla\left(\psi_{k} \varphi\right) . \nabla u+$ $\nabla^{*}\left(\rho u \nabla\left(\psi_{k} \varphi\right)\right) \in \mathscr{H}^{-1}(X)$.

Pour tout $v \in L^{2}(X), \lim _{k \rightarrow \infty} \psi_{k} v=v$ dans $L^{2}(X)$. Puisqu'il existe une constante $C>0$ telle que $|\varphi(x)| \leq C\langle x\rangle$ pour tout $x \in X$, on a aussi $\lim _{k \rightarrow \infty}\left|\nabla \varphi_{k}\right| \varphi v=0$ dans $L^{2}(X)$. On en déduit que $\lim _{k \rightarrow \infty} g_{k}=\varphi f-\rho \nabla \varphi \cdot \nabla u+$ $\nabla^{*}(\rho u \nabla \varphi)=: g$ dans $\mathscr{H}^{-1}(X)$, donc $\varphi u=(H-\lambda)^{-1} g \in \mathscr{H}^{1}(X)$.

Pour conclure, il suffit de choisir

$$
\begin{aligned}
T(\varphi, \lambda):= & -\left[(H-\lambda)^{-1} \rho(\nabla \varphi)\right] \cdot\left[\nabla(H-\lambda)^{-1}\right] \\
& +(H-\lambda)^{-1} \nabla^{*}\left[(\rho \nabla \varphi)(H-\lambda)^{-1}\right] .
\end{aligned}
$$

La dernière propriété résulte du fait que l'opérateur de multiplication par une 
fonction $\psi \in C(X)$ est compact de $\mathscr{H}^{1}(X)$ dans $L^{2}(X)$ et de $L^{2}(X)$ dans $\mathscr{H}^{-1}(X)$ lorsque $\lim _{|x| \rightarrow \infty} \varphi(x)=0$.

Q.E.D.

Lemme 3.2. Soient $f \in C_{0}^{\infty}(\mathbb{R})$ et $\varphi \in C^{1}(X)$ telle que $\nabla \varphi$ soit borné. Alors $f(H) \in \mathscr{B}\left(\mathscr{H}^{-1}(X), \mathscr{H}^{1}(X)\right)$ et il existe un opérateur $T=T(f, \varphi) \in$ $\mathscr{B}\left(\mathscr{H}^{-1}(X), \mathscr{H}^{1}(X)\right)$ tel que pour tout $u \in \mathscr{H}^{-1}(X)$ vérifiant $\varphi u \in \mathscr{H}^{-1}(X)$ on ait

$$
\varphi f(H) u=f(H)(\varphi u)+T u .
$$

De plus, si $\lim _{|x| \rightarrow \infty}|\nabla \varphi(x)|=0$, alors $T \in \mathscr{K}\left(\mathscr{H}^{-1}(X), \mathscr{H}^{1}(X)\right)$.

Démonstration. D'après le théorème 6.1 .4 de [2], pour tout $r \geq 2$ on a dans $\mathscr{B}\left(L^{2}(X)\right)$

$$
\begin{aligned}
f(H)= & \sum_{0 \leq j \leq r-1} \frac{1}{\pi j !} \int f^{(J)}(\lambda) \operatorname{Im}\left[i^{j}(H-\lambda-i)^{-1}\right] d \lambda \\
& +\frac{1}{\pi(r-1) !} \int_{0}^{1} \tau^{r-1}\left\{\int f^{(r)}(\lambda) \operatorname{Im}\left[i^{r}(H-\lambda-i \tau)^{-1}\right] d \lambda\right\} d \tau,
\end{aligned}
$$

où $i=\sqrt{-1}$ et, pour $B \in \mathscr{B}\left(L^{2}(X)\right)$, on pose $\operatorname{Im} B:=(2 i)^{-1}\left(B-B^{*}\right)$. D'après le lemme 3.1 et la relation (3.3), on aura $\varphi(H-\lambda-i \tau)^{-1} u=(H-\lambda-i \tau)^{-1}(\varphi u)$ $+T(\varphi, \lambda, \tau) u$ pour tous $\lambda, \tau \in \mathbb{R}, 0<|\tau| \leq 1$, où $T(\varphi, \lambda, \tau):=(H-\lambda-i \tau)^{-1}$ $K_{\varphi}(H-\lambda-i \tau)^{-1}$, avec $K_{\varphi} v:=-\rho \nabla \varphi \cdot \nabla v+\nabla^{*}(\rho v \nabla \varphi)$ si $v \in \mathscr{H}^{1}(X)$. On voit que l'opérateur $K_{\varphi}$ est borné (et même compact si $\lim _{|x| \rightarrow \infty}|\nabla \varphi(x)|=0$ ) de $\mathscr{H}^{1}(X)$ dans $\mathscr{H}^{-1}(X)$.

On vérifie aussi qu'il existe une constante $C_{0}>0$ telle que $\left.\|(H-\lambda-i \tau)^{-1}\right) \|_{\mathscr{B}\left(\mathscr{H}^{-1}(X), \mathscr{H}^{1}(X)\right)} \leq C_{0}|\tau|^{-1}\left(1+\lambda^{2}\right)^{1 / 2}$ si $\lambda, \tau \in \mathbb{R}$ et $0<|\tau| \leq 1$. On en déduit qu'il existe une autre constante $C>0$ telle que, pour les mêmes valeurs de $\lambda$ et de $\tau$, l'opérateur $T(\varphi, \lambda, \tau)$ soit borné (et même compact si $\left.\lim _{|x| \rightarrow \infty}|\nabla \varphi(x)|=0\right)$ de $\quad \mathscr{H}^{-1}(X)$ dans $\mathscr{H}^{1}(X)$ et que $\|T(\varphi, \lambda, \tau)\|_{\mathscr{B}\left(\mathscr{H}^{-1}(X), \mathscr{H}^{1}(X)\right)} \leq C|\tau|^{-2}\left(1+\lambda^{2}\right)$.

Les propriétés de l'énoncé résultent alors de (3.5).

Q.E.D.

Nous rappelons maintenant la définition d'une partition de l'unité de Ruelle-Simon suivant [1]. Pour tout $Y \in \mathscr{L}$, on désigne par $\pi_{Y}$ la projection orthogonale de $X$ sur $Y$. Si $x \in X$ et $Y \in \mathscr{L}_{1}$ on définit

$$
[x]_{Y}:=\min _{Z \in \mathscr{L} \backslash \mathscr{L}(Y)}\left|\pi_{Z} x\right|=\operatorname{dist}\left(x, \bigcup_{Z \in \mathscr{L} \backslash \mathscr{L}(Y)} Z^{\perp}\right)
$$

Remarquons que $\left\{x \in Y^{\perp} ;[x]_{Y} \neq 0\right\}=Y^{\perp} \backslash \bigcup_{Z \in \mathscr{L} \backslash \mathscr{L}(Y)} Z^{\perp}$ est un cône ouvert de $Y^{\perp}$ et dense. 
Pour tout $d \in[0,1]$ et tout $Y \in \mathscr{L}_{1}$ on note $\Gamma_{Y}(d):=\left\{x \in X ;[x]_{Y}>d|x|\right\}$.

Lemme 3.3. (a) $\Gamma_{Y}(d)$ est un cône ouvert dans $X$.

(b) Si $0 \leq d_{1}<d_{2}<1$, alors $\Gamma_{Y}\left(d_{2}\right) \subset \Gamma_{Y}\left(d_{1}\right)$.

(c) $\Gamma_{Y}(0)=\bigcup_{0 \leq d<1} \Gamma_{Y}(d)$.

(d) Si $d \geq 0$ est assez petit, on a $X \backslash\{0\}=\bigcup_{Y \in \mathscr{L}_{1}} \Gamma_{Y}(d)$.

(e) Si $M$ est un cône fermé de $X$, tel que $M \backslash\{0\} \subset \Gamma_{Y}(0)$, il existe une constante $C>0$ telle que $|x| \leq C\left|\pi_{Z} x\right|$ pour tout $x \in M$ et tout $Z \in \mathscr{L} \backslash \mathscr{L}(Y)$.

(f) Si $K$ est un compact de $X, Y \in \mathscr{L}_{1}$ et $\Gamma$ est un cône fermé de $Y^{\perp}$, $\Gamma \backslash\{0\} \subset Y^{\perp} \backslash \bigcup_{Z \in \mathscr{L} \backslash \mathscr{L}(Y)} Z$, alors il existe des constantes $C>0$ et $d \in(0,1)$ telles que pour tout $y \in \Gamma$, qui vérifie $|y|>C$, on ait $K+\{y\} \subset \Gamma_{Y}(d)$.

Démonstration. Les propriétés (a)-(e) résultent des lemmes 2.2 .2 et 2.2.3 de [1] et (f) du lemme 5.7 de [13].

Q.E.D.

Définition 3.4. On appelle $\mathscr{L}$-partition de l'unité sur $X$, une famille $\left\{\chi_{Y}\right\}_{Y \in \mathscr{L}_{1}}$ telle que:

(a) $\chi_{Y} \in C^{\infty}(X, \mathbb{R})$,

(b) supp $\chi_{Y} \cap\{x \in X ;|x| \geq 1\} \subset \Gamma_{Y}(0)$,

(c) $\chi_{Y}(x)=\chi_{Y}(x /|x|)$ pour tout $x \in X$ avec $|x| \geq 1$,

(d) $\sum_{Y \in \mathscr{L}_{1}} \chi_{Y}^{2}=1$ sur $X$.

Remarque 3.5. (a) L'existence d'une $\mathscr{L}$-partition de l'unité sur $X$ résulte du lemme 2.2.5 de [1].

(b) D'après le lemme 3.3 (e), il existe une constante $C>0$ telle que pour tous $Y \in \mathscr{L}_{1}$ et $Z \in \mathscr{L} \backslash \mathscr{L}(Y)$ on ait

$$
\langle x\rangle \leq C\left\langle\pi_{Z} x\right\rangle \text { pour tout } x \in \operatorname{supp} \chi_{Y} .
$$

(c) D'après le lemme 2.5. 2 (b) de [1] et l'hypothèse III-(i), pour tout $Y \in$ $\mathscr{L}_{1}, \chi_{Y} I_{Y} \in \mathscr{K}\left(\mathscr{H}^{1}(X), \mathscr{H}^{-1}(X)\right)$.

Lemme 3.6. Pour tous $Y \in \mathscr{L}_{1}$ et $\lambda \in \mathbb{C} \backslash \sigma(H)$ on a

$$
\chi_{Y}\left[(H-\lambda)^{-1}-\left(H_{Y}-\lambda\right)^{-1}\right] \in \mathscr{K}\left(\mathscr{H}^{-1}(X), L^{2}(X)\right)
$$

et

$$
\left[(H-\lambda)^{-1}-\left(H_{Y}-\lambda\right)^{-1}\right] \chi_{Y} \in \mathscr{K}\left(\mathscr{H}^{-1}(X), L^{2}(X)\right) .
$$

Démonstration. Désignons par $R_{Y} \in \mathscr{B}\left(\mathscr{H}^{-1}(X), \mathscr{H}^{1}(X)\right)$ l'opérateur défini par le côté gauche de (3.7). D'après le lemme 2.5.1 de [1], $I_{Y}$ appartient à $\mathscr{B}\left(\mathscr{H}^{1}(X), \mathscr{H}^{-1}(X)\right)$ et alors on a $R_{Y}=R_{Y}^{\prime}+R_{Y}^{\prime \prime}$, où $R_{Y}^{\prime}:=\chi_{Y}(H-\lambda)^{-1}$. $M_{Y}\left(H_{Y}-\lambda\right)^{-1}$ avec $M_{Y}:=-\nabla^{*}\left(\rho-\rho_{Y}\right) \nabla \in \mathscr{B}\left(\mathscr{H}^{1}(X), \mathscr{H}^{-1}(X)\right)$ et $R_{Y}^{\prime \prime}:=$ $-\chi_{Y}(H-\lambda)^{-1} I_{Y}\left(H_{Y}-\lambda\right)^{-1}$. D'après l'hypothèse II et la remarque $3.5(\mathrm{~b})$, 
\langle\rangle$^{\theta} \chi_{Y} M_{Y} \in \mathscr{B}\left(\mathscr{H}^{1}(X), \mathscr{H}^{-1}(X)\right)$, donc, en utilisant le lemme 3.1, \langle\rangle$^{\theta} R_{Y}^{\prime} \in$ $\mathscr{B}\left(\mathscr{H}^{-1}(X), \mathscr{H}^{1}(X)\right)$ et alors $R_{Y}^{\prime} \in \mathscr{K}\left(\mathscr{H}^{-1}(X), L^{2}(X)\right)$.

D'autre part, d'après la remarque 3.5 (c) et le même lemme 3.1 , on a $R_{Y}^{\prime \prime} \in$ $\mathscr{K}\left(\mathscr{H}^{-1}(X), \mathscr{H}^{1}(X)\right)$, d'où l'on déduit (3.7). On prouve de la même façon $\left(3.7^{\prime}\right)$.

Q.E.D.

Lemme 3.7. Pour tous $Y \in \mathscr{L}_{1}$ et $f \in C_{0}^{\infty}(\mathbb{R})$ on a

$$
\chi_{Y}\left[f(H)-f\left(H_{Y}\right)\right] \in \mathscr{K}\left(\mathscr{H}^{-1}(X), L^{2}(X)\right)
$$

et

$$
\left[f(H)-f\left(H_{Y}\right)\right] \chi_{Y} \in \mathscr{K}\left(\mathscr{H}^{-1}(X), L^{2}(X)\right) .
$$

Démonstration. On peut représenter l'opérateur défini par le côté gauche de (3.8) en utilisant (3.5) et alors, d'après le lemme 3.6, tout revient à estimer la norme de l'opérateur

$$
\begin{aligned}
S_{Y}(\lambda, \tau) & :=\varphi\left[(H-\lambda-i \tau)^{-1}-\left(H_{Y}-\lambda-i \tau\right)^{-1}\right] \\
& =\left[(H-\lambda-i \tau)^{-1} \varphi Q_{Y}+T_{Y}(\lambda, \tau) Q_{Y}\right]\left(H_{Y}-\lambda-i \tau\right)^{-1}
\end{aligned}
$$

en fonction de $\lambda \in \mathbb{R}$ et $\tau \in(0,1]$, où $\varphi:=\langle\rangle^{\theta} \chi_{Y}, Q_{Y}:=-\nabla^{*}\left(\rho-\rho_{Y}\right) \nabla-I_{Y}$ et $T_{Y}(\lambda, \tau):=(H-\lambda-i \tau)^{-1} K_{\varphi}(H-\lambda-i \tau)^{-1}$, tandis que $K_{\varphi}$ a été défini au courant de la démonstration du lemme 3.2. On aura donc $\left\|S_{Y}(\lambda, \tau)\right\|_{\mathscr{B}\left(\mathscr{H}^{-1}(X) \mathscr{H}^{1}(X)\right)} \leq C|\tau|^{-3}\left(1+\lambda^{2}\right)^{3 / 2}$ avec $C$ constante positive. Il en résulte (3.8). Pour vérifier $\left(3.8^{\prime}\right)$ on utilise un raisonnement similaire. Q.E.D.

Proposition 3.8. Pour tout $f \in C_{0}^{\infty}(\mathbb{R})$ on a

$$
f(H)^{2}-\sum_{Y \in \mathscr{L}_{1}} \chi_{Y} f\left(H_{Y}\right)^{2} \chi_{Y} \in \mathscr{K}\left(\mathscr{H}^{-1}(X), \mathscr{H}^{1}(X)\right) .
$$

Démonstration. Il suffit de remarquer l'identité

$$
\begin{aligned}
f(H)^{2}= & \sum_{Y \in \mathscr{L}_{1}} f(H) \chi_{Y}^{2} f(H) \\
= & \sum_{Y \in \mathscr{L}_{1}}\left\{\chi_{Y} f\left(H_{Y}\right)^{2} \chi_{Y}+\left[f(H), \chi_{Y}\right] \chi_{Y} f(H)+\chi_{Y} f(H)\left[\chi_{Y}, f(H)\right]\right. \\
& \left.+\chi_{Y}\left[f(H)-f\left(H_{Y}\right)\right] f(H) \chi_{Y}+\chi_{Y} f\left(H_{Y}\right)\left(f(H)-f\left(H_{Y}\right)\right] \chi_{Y}\right\},
\end{aligned}
$$

utiliser les lemmes 3.2 et 3.7 , et noter que, par dualité, l'opérateur défini par le côté gauche de (3.8) appartient à $\mathscr{K}\left(L^{2}(X), \mathscr{H}^{1}(X)\right)$ Q.E.D.

Démonstration des propriétés (a) et (c) du théorème 1.2

(a) Prouvons d'abord l'inclusion $\sigma_{e s s}(H) \subset \bigcup_{Y \in \mathscr{L}_{1}} \sigma_{e s s}\left(H_{Y}\right)$. Si $\mu \in \mathbb{R}, \mu \notin$ $\bigcup_{Y \in \mathscr{L}_{1}} \sigma_{e s s}\left(H_{Y}\right)$ et $f \in C_{0}^{\infty}(\mathbb{R}), \quad f(\mu) \neq 0, \operatorname{supp} f \cap\left[\bigcup_{Y \in \mathscr{L}_{1}} \sigma_{e s s}\left(H_{Y}\right)\right]=\varnothing$, alors 
$f\left(H_{Y}\right) \in \mathscr{K}\left(L^{2}(X)\right)$ pour tout $Y \in \mathscr{L}_{1}$. D'après (3.10), $f(H) \in \mathscr{K}\left(L^{2}(X)\right)$, donc $\mu \notin \sigma_{\text {ess }}(H)$.

Pour l'inclusion réciproque on utilise la variante suivante du critère de Weyl: $\mu \in \sigma_{e s s}(H)$ si et seulement s'il existe une suite $\left\{u_{j}\right\}_{j \geq 1} \subset \mathscr{H}^{1}(X),\left\|u_{j}\right\|=1$ telle qu'elle converge faiblement vers zéro dans $L^{2}(X)$ et $\lim _{j \rightarrow \infty}(H-\mu) u_{j}=0$ dans $\mathscr{H}^{-1}(X)$. Soit alors $Y \in \mathscr{L}_{1}$ et $\mu \in \sigma_{\text {ess }}\left(H_{Y}\right)$; étant donné que $C_{0}^{\infty}(X)$ est dense dans $\mathscr{H}^{1}(X)$, on peut trouver une suite $\left\{u_{j}\right\}_{j \geq 1} \subset C_{0}^{\infty}(X)$ qui ait les propriétés ci-dessus relativement à $H_{Y}$. Cette suite sera bornée dans $\mathscr{H}^{1}(X)$ car, pour tout $\lambda \in \mathbb{C} \backslash \sigma(H), u_{j}=(H-\lambda)^{-1}(H-\mu) u_{j}-(\lambda-\mu)(H-\lambda)^{-1} u_{j}$.

D'après le lemme 3.3 (f), il existe une suite $\left\{x^{j}\right\}_{j \geq 1} \subset Y^{\perp}$ et $d \in(0,1)$ tels que pour tout $j \geq 1, \operatorname{supp} u_{j}+\left\{x^{j}\right\} \subset \Gamma_{Y}(d) \cap\{x \in X ;|x| \geq j\}$. Désignons par $T_{j}$ l'opérateur de translation sur $L^{2}(X)$ de vecteur $x^{j}$ et posons $v_{j}:=T_{j} u_{j}$.

Alors $v_{j} \in C_{0}^{\infty}(X),\left\|v_{j}\right\|_{L^{2}(X)}=1$ et $\operatorname{supp} v_{j} \subset \Gamma_{Y}(d) \cap\{x \in X ;|x| \geq j\}$, donc, en particulier, $\lim _{j \rightarrow \infty} v_{j}=0$ pour la topologie faible de $L^{2}(X)$. La suite $\left\{v_{j}\right\}_{j \geq 1}$ est bornée dans $\mathscr{H}^{1}(X)$ et $\operatorname{supp} v_{j} \subset\{x \in X .|x| \geq j\}$, donc $\lim _{j \rightarrow \infty} v_{j}=0$ pour la topologie faible de $\mathscr{H}^{1}(X)$. Il reste à prouver que $\lim _{j \rightarrow \infty}(H-\mu) v_{j}=0$ dans $\mathscr{H}^{-1}(X)$. On note que $(H-\mu) v_{j}=T_{j}\left(H_{Y}-\mu\right) u_{j}+\left(H-H_{Y}\right) v_{j}$, où le premier terme du côté droit tend vers zéro dans $\mathscr{H}^{-1}(X)$ si $j \rightarrow \infty$ et $H-H_{Y}=$ $\nabla^{*}\left(\rho-\rho_{Y}\right) \nabla+I_{Y}$.

Soit une fonction $\chi$ possédant les propriétés (a), (b), (c) de la définition 3.4 et telle que $\chi=1$ sur $\Gamma_{Y}(d) \cap\{x \in X ;|x| \geq 1\}$. Alors $I_{Y} v_{j}=\chi I_{Y} v_{j}$ et d'après la remarque 3.5 (c), $\lim _{j \rightarrow \infty} I_{Y} v_{j}=0$ dans $\mathscr{H}^{-1}(X)$. Finalement,

$$
\left\|\nabla^{*}\left(\rho-\rho_{Y}\right) \nabla v_{j}\right\|_{\mathscr{H}^{-1}(X)}=\sup _{\varphi \in C_{0}^{\infty}(X), \varphi \neq 0}\|\varphi\|_{\mathscr{H}^{1}(X)}^{-1}\left|\int_{X} \chi\left(\rho-\rho_{Y}\right) \nabla v_{j} . \nabla \bar{\varphi} d x\right|
$$

et par l'hypothèse II et la remarque 3.5 (b), il existe une constante $C>0$ telle que sur supp $v_{j}$ l'on vérifie $\left|\chi\left(\rho-\rho_{Y}\right)(x)\right| \leq C\langle x\rangle^{-\theta} \leq C j^{-\theta}$ pour tous $x \in X$ et $j \geq 1$. Alors $\lim _{j \rightarrow \infty} \nabla^{*}\left(\rho-\rho_{Y}\right) \nabla v_{j}=0$ dans $\mathscr{H}^{-1}(X)$.

(c) C'est évident, car $H(\rho, 0) \geq 0$ et alors $\sigma_{e s s}(H(\rho, 0)) \subset \sigma(H(\rho, 0)) \subset$ $[0, \infty)=\sigma_{\text {ess }}\left(H\left(\rho_{0}, 0\right)\right) \subset \sigma_{\text {ess }}(H(\rho, 0))$, où pour la dernière inclusion on utilise (a).

Q.E.D.

\section{§4. L’Opérateur Conjugué}

Dans cette section on suppose que toutes les hypothèses I-IV sont vérifiées.

Soit d'abord $Z \in \mathscr{L}_{m}$. On choisit $\psi \in C_{0}^{\infty}(Z)$ fonction réelle, positive, paire (donc sa transformée de Fourier $\hat{\psi}$ sera elle aussi réelle), telle que $\hat{\psi}$ soit positive, $\hat{\psi}(0)=1$ et $\operatorname{supp} \psi \subset\{z \in Z ;|z| \leq 1\}$. Pour la construire, on considère une autre fonction $\psi_{0} \in C_{0}^{\infty}(Z)$ réelle, positive, paire, telle que $\hat{\psi}_{0}(0)=1$ et 
$\operatorname{supp} \psi_{0} \subset\{z \in Z ;|z| \leq 1 / 2\}$ et on définit $\psi:=\psi_{0} * \psi_{0}$, où $*$ désigne l'opération de convolution. Pour $\varepsilon \in(0,1]$ (qui sera précisé ultérieurement en fonction de l'intervalle spectral considéré) on définit une nouvelle fonction $\psi_{\varepsilon} \in C_{0}^{\infty}(Z)$ par $\psi_{\varepsilon}(z):=\varepsilon^{-\operatorname{dim} Z} \psi(z / \varepsilon)$.

On introduit l'opérateur $R=R^{Z}: \mathscr{D}^{\prime}(Z) \rightarrow \mathscr{D}^{\prime}(Z)$ défini par

$$
R u:=-i\left(z \cdot \nabla_{z}\right) u, \quad u \in \mathscr{D}^{\prime}(Z),
$$

où $\nabla_{z}$ désigne l'opérateur gradient dans $Z$ et soit $R^{*}$ l'adjoint formel de $R$. On peut maintenant définir l'opérateur $A^{Z}=A_{\varepsilon}^{Z}: \mathscr{D}^{\prime}(Z) \rightarrow \mathscr{E}(Z)$, linéaire et continu, par

$$
\begin{aligned}
A^{Z} u & :=\frac{1}{2}\left[R\left(\psi_{\varepsilon} * u\right)+\psi_{\varepsilon} *\left(R^{*} u\right)\right] \\
& =R\left(\psi_{\varepsilon} * u\right)-\frac{1}{2}\left(R \psi_{\varepsilon}\right) * u, \quad u \in \mathscr{D}^{\prime}(Z) .
\end{aligned}
$$

Remarque 4.1. On voit que pour tout $u \in \mathscr{D}^{\prime}(Z), \lim _{\varepsilon \searrow 0} A_{\varepsilon}^{Z} u=\frac{1}{2}\left(R u+R^{*} u\right)$ dans $\mathscr{D}^{\prime}(Z)$, où $\frac{1}{2}\left(R+R^{*}\right)$, considéré en tant qu'opérateur auto-adjoint sur $L^{2}(Z)$, de domaine essentiel $C_{0}^{\infty}(Z)$, est le générateur des dilatations sur $Z$.

Remarque 4.2. On peut aussi bien considérer $R$ comme un opérateur sur $\mathscr{D}^{\prime}(Z ; Z \otimes \mathbb{C})$ et $A^{Z}: \mathscr{D}^{\prime}(Z ; Z \otimes \mathbb{C}) \rightarrow \mathscr{E}(Z ; Z \otimes \mathbb{C})$.

Lemme 4.3. (a) Pour tout $u \in \mathscr{D}^{\prime}(Z)$, supp $A^{Z} u \subset\{z \in Z ; \operatorname{dist}(z, \operatorname{suppu}) \leq$ $\varepsilon\}$.

(b) Pour tout $u \in \mathscr{D}^{\prime}(Z)$ on a $\nabla_{z} A^{Z} u=A^{Z} \nabla_{z} u+F^{Z} u$, où $F^{Z}: \mathscr{D}^{\prime}(Z) \rightarrow$ $\mathscr{E}(Z ; Z \otimes \mathbb{C})$ est défini par

$$
F^{Z} u:=-i \nabla_{z}\left(\psi_{\varepsilon} * u\right), \quad u \in \mathscr{D}^{\prime}(Z) .
$$

(c) L'espace $\mathscr{S}(Z)$ est invariant par rapport à l'opérateur $A^{Z}$.

(d) L'opérateur $A^{Z}$, de domaine $\mathscr{S}(Z)$, est essentiellement auto-adjoint sur $L^{2}(Z)$ (on désignera par le même symbole l'opérateur auto-adjoint correspondant).

(e) Pour tous $\tau, s \in \mathbb{R}$, les espaces $\mathscr{S}(Z)$ et $\mathscr{H}^{s}(Z)$ sont invariants par rapport à $e^{i \tau A^{Z}}$. De plus, pour tout $s \in \mathbb{R}$, il existe des constantes $M \geq 1$ et $\omega \geq 0$ telles que

$$
\left\|e^{i \tau A^{Z}}\right\|_{\mathscr{B}\left(\mathscr{H}{ }^{s}(Z)\right)} \leq M e^{\omega|\tau|}, \quad \tau \in \mathbb{R} .
$$

(f) Si $u \in L^{2}(Z)$ et $\langle z\rangle u \in L^{2}(Z)$, alors $u \in D\left(A^{Z}\right)$.

Démonstration. Les propriétés (a), (b) et (c) résultent directement de la définition de $A^{Z}$, tandis que (d) et (e) du lemme 1.3 de [7].

Si $u$ vérifie les hypothèses du (f), on peut trouver une suite $\left\{\varphi_{j}\right\}_{j \geq 1} \subset$ $C_{0}^{\infty}(Z)$ telle que $\lim _{j \rightarrow \infty} \varphi_{j}=u$ et $\lim _{j \rightarrow \infty}\langle z\rangle \varphi_{j}=\langle z\rangle u$ dans $L^{2}(Z)$. On a aussi 


$$
A^{Z} v=\psi_{\varepsilon} *\left(R^{*} v\right)+\frac{1}{2}\left(R \psi_{\varepsilon}\right) * v, \quad v \in \mathscr{D}^{\prime}(Z)
$$

d'où l'on déduit que $\lim _{j \rightarrow \infty} A^{Z} \varphi_{j}=A^{Z} u$ dans $L^{2}(Z)$ et donc $u \in D\left(A^{Z}\right)$. Q.E.D.

Pour tout $Y \in \mathscr{L} \backslash\{0\}$ on définit les opérateurs $A^{Y}=A_{\varepsilon}^{Y}: \mathscr{D}^{\prime}(Y) \rightarrow \mathscr{D}^{\prime}(Y)$ (ou éventuellement $\quad A^{Y}: \mathscr{D}^{\prime}(Y ; Y \otimes \mathbb{C}) \rightarrow \mathscr{D}^{\prime}(Y ; Y \otimes \mathbb{C})$ ) et $A_{Y}=A_{\varepsilon, Y}$ : $\mathscr{D}^{\prime}(X) \rightarrow \mathscr{D}^{\prime}(X)$ (ou bien $A_{Y}: \mathscr{D}^{\prime}(X ; X \otimes \mathbb{C}) \rightarrow \mathscr{D}^{\prime}(X ; X \otimes \mathbb{C})$ ) par

$$
A^{Y}:=\sum_{Z \in \mathscr{L}_{m}(Y)}\left(A^{Z} \otimes 1_{Y \ominus Z}\right), \quad A_{Y}:=A^{Y} \otimes 1_{Y^{\perp}} .
$$

En particulier, on définit $A=A_{\varepsilon}:=A^{X}=A_{X}$.

Lemme 4.4. (a) Les espaces $\mathscr{E}^{\prime}(X)$ et $\mathscr{S}(X)$ sont invariants par rapport à l'opérateur $A$.

(b) Pour tout $u \in \mathscr{D}^{\prime}(X)$ on a $\nabla A u=A \nabla u+F u$, où $F: \mathscr{D}^{\prime}(X) \rightarrow$ $\mathscr{D}^{\prime}(X ; X \otimes \mathbb{C})$ est défini par $F:=\sum_{Z \in \mathscr{L}_{m}} F_{Z}$ avec $F_{Z}: \mathscr{D}^{\prime}(X) \rightarrow \mathscr{D}^{\prime}(X ; X \otimes \mathbb{C})$,

$$
F_{Z} u:=i_{Z}\left(F^{Z} \otimes 1_{Z^{\perp}}\right) u, \quad u \in \mathscr{S}(X),
$$

où $i_{Z}$ est l'injection canonique de $Z$ dans $X$ (prolongée en tant qu'injection de $Z \otimes \mathbb{C}$ dans $X \otimes \mathbb{C}$.

(c) L'opérateur $A$, de domaine $\mathscr{S}(X)$, est essentiellement auto-adjoint sur $L^{2}(X)$ (on désigne encore par A l'unique extension auto-adjointe de cet opérateur).

(d) Pour tous $\tau, s \in \mathbb{R}$, les espaces $\mathscr{S}(X)$ et $\mathscr{H}^{s}(X)$ sont invariants par rapport à $e^{i \tau A}$. De plus, pour tout $s \in \mathbb{R}$, il existe des constantes $M \geq 1$ et $\omega \geq 0$ telles que

$$
\left\|e^{i \tau A}\right\|_{\mathscr{B}\left(\mathscr{P}^{s}(X)\right)} \leq M e^{\omega|\tau|}, \quad \tau \in \mathbb{R} .
$$

(e) Si $u \in L^{2}(X)$ et $\langle\rangle . u \in L^{2}(X)$, alors $u \in D(A)$.

Démonstration. Les propriétés (a)-(c) et (e) résultent du lemme 4.3. La première partie du (d) résulte du lemme 1.3 de [7] et l'inégalité (4.8) de la proposition 3.2.2 de [2].

Q.E.D.

Pour étudier la régularité de $H$ par rapport à $A$ on aura besoin de quelques propriétés auxiliaires. Remarquons d'abord que, d'après le lemme 4.4 (d) et la section 4 de [4], pour tout $s \geq 0,\left(\mathscr{H}^{s}(X), L^{2}(X)\right)$ est un couple de Friedrichs et que $\left\{e^{i \tau A}\right\}_{\tau \in \mathbb{R}}$ est un groupe unitaire dans ce couple.

Lemme 4.5. Soient $\theta \in(0,1], s \geq 0$ et $T \in \mathscr{B}\left(\mathscr{H}^{s}(X), \mathscr{H}^{-s}(X)\right)$ symétrique, tels que \langle\rangle$^{\theta} T \in \mathscr{B}\left(\mathscr{H}^{s}(X), \mathscr{H}^{-s}(X)\right)$. Alors $T$ appartient à $C^{\theta}\left(A ; \mathscr{H}^{s}(X)\right.$, $\left.\mathscr{H}^{-s}(X)\right)$. 
Démonstration. La démarche de la première partie de la preuve de la proposition 7.5.7 de [2] permet d'estimer la norme du commutateur $\left[e^{i \tau A}, T\right]$ dans $\mathscr{B}\left(\mathscr{H}^{s}(X), \mathscr{H}^{-s}(X)\right)$ pour $\tau \in\left[0, \tau_{0}\right], \tau_{0}>0$ assez petit. Plus exactement, d'après [2] et l'inégalité (4.8), pour tout $t \in \mathbb{R}$, il existe $\tau_{0}$ assez petit, tel que l'on ait:

(a) $\sup _{0 \leq \tau \leq \tau_{0}}\left(\left\|(\tau A+2 i)^{-1}\right\|_{\mathscr{B}\left(\mathscr{H}^{t}(X)\right)}+\left\|\tau A(\tau A+2 i)^{-1}\right\|_{\mathscr{B}\left(\mathscr{H}^{t}(X)\right)}\right)<\infty$,

(b) $\mathrm{Si} f: \mathbb{R} \rightarrow \mathbb{C}$ est une fonction définie par l'égalité $\sin \tau=$ $\tau(\tau+i)^{-1} f(\tau), \tau \in \mathbb{R}$, alors $\sup _{0 \leq \tau \leq \tau_{0}}\|f(\tau A)\|_{\mathscr{B}\left(\mathscr{H}^{t}(X)\right)}<\infty$.

Remarquons aussi les propriétés suivantes:

(c) $A\langle.\rangle^{-1} \in \mathscr{B}\left(\mathscr{H}^{t}(X)\right)$ pour tout $t \in \mathbb{R}$,

(d) Pour tout $t \in \mathbb{R}$, l'opérateur $\tau A(\tau A+2 i)^{-1}(\tau\langle\rangle+1.)(\tau\langle.\rangle)^{-1}=$ $\tau A(\tau A+2 i)^{-1}+(\tau A+2 i)^{-1} A\langle\rangle^{-1}$ est borné sur $\mathscr{H}^{t}(X)$, uniformément par rapport à $\tau \in\left(0, \tau_{0}\right]$,

(e) L'opérateur de multiplication par $(\tau\langle.\rangle)^{1-\theta}(\tau\langle\cdot\rangle+1)^{-1}$ est dans $\mathscr{B}\left(\mathscr{H}^{t}(X)\right)$, uniformément par rapport à $\tau \in\left[0, \tau_{0}\right]$,

(f) Il existe une constante $C_{0}>0$ telle que la norme de l'opérateur $\tau\langle.\rangle(\tau\langle.\rangle+1)^{-1} T$ dans $\mathscr{B}\left(\mathscr{H}^{s}(X), \mathscr{H}^{-s}(X)\right)$ soit majorée par $C_{0} \tau^{\theta}$ pour tout $\tau \in\left[0, \tau_{0}\right]$

Pour finir la démonstration on écrit $\left[e^{i \tau A}, T\right]=\left(e^{i \tau A}-1\right) T-T\left(e^{i \tau A}-1\right)$ et $e^{i \tau A}-1=2 i e^{i \tau A / 2} \sin \frac{\tau A}{2}$. D'après les propriétés (d) et (f), il existe une constante $C_{1}>0$ telle que $\left\|\tau A(\tau A+2 i)^{-1} T\right\|_{\mathscr{B}\left(\mathscr{H}^{s}(X), \mathscr{H}^{-s}(X)\right)} \leq C_{1} \tau^{\theta}$ si $\tau \in\left[0, \tau_{0}\right]$. Utilisant aussi les propriétés (a) et (b), l'identité ci-dessus ainsi que (4.8), on déduit l'existence d'une autre constante $C_{2}>0$ telle que $\left\|\left(e^{i \tau A}-1\right) T\right\|_{\mathscr{B}\left(\mathscr{H}^{s}(X), \mathscr{H}^{-s}(X)\right)} \leq C_{2} \tau^{\theta}$ si $\tau \in\left[0, \tau_{0}\right]$. On a une estimation similaire pour le terme $T\left(e^{i \tau A}-1\right)$, donc il existe une constante $C>0$ telle que $\left\|\left[e^{i \tau A}, T\right]\right\|_{\mathscr{B}\left(\mathscr{H}^{s}(X), \mathscr{H}^{-s}(X)\right)} \leq C \tau^{\theta}$ pour $\tau \in\left[0, \tau_{0}\right]$, d'où $T \in C^{\theta}(A ;$ $\left.\mathscr{H}^{s}(X), \mathscr{H}^{-s}(X)\right)$.

Q.E.D.

Lemme 4.6. Soient $\theta \in(0,1], s \geq 0, Y \in \mathscr{L}, M \in C^{\theta}\left(A^{Y} ; \mathscr{H}^{s}(Y), \mathscr{H}^{-s}(Y)\right)$ et $N \in C^{\theta}\left(A^{Y^{\perp}} ; L^{2}\left(Y^{\perp}\right)\right)$. Alors l'opérateur $M \otimes N$ (bien défini de $\mathscr{S}(X)$ dans $\left.\mathscr{S}^{\prime}(X)\right)$ appartient à $C^{\theta}\left(A ; \mathscr{H}^{s}(X), \mathscr{H}^{-s}(X)\right)$.

Démonstration. D'après le lemme 2.5 .1 de [1], pour tout $M \in$ $\mathscr{B}\left(\mathscr{H}^{s}(Y), \mathscr{H}^{-s}(Y)\right)$ et tout $N \in \mathscr{B}\left(L^{2}\left(Y^{\perp}\right)\right)$, on a $M \otimes N \in \mathscr{B}\left(\mathscr{H}^{s}(X), \mathscr{H}^{-s}(X)\right)$ et $\|M \otimes N\|_{\mathscr{B}\left(\mathscr{H}^{s}(X), \mathscr{H}^{-s}(X)\right)} \leq\|M\|_{\mathscr{B}\left(\mathscr{H}^{s}(Y), \mathscr{H}^{-s}(Y)\right)}\|N\|_{\mathscr{B}\left(L^{2}\left(Y^{\perp}\right)\right)}$.

Grâce à l'hypothèse IV, on a $e^{i \tau A}=e^{i \tau A^{Y}} \otimes e^{i \tau A^{Y^{\perp}}}$, donc $\left[e^{i \tau A}, M \otimes N\right]=$ $\left[e^{i \tau A^{Y}}, M\right] \otimes e^{i \tau A^{Y^{\perp}}} N+M e^{i \tau A^{Y}} \otimes\left[e^{i \tau A^{Y^{\perp}}}, N\right]$. Il suffit d'utiliser les hypothèses et l'inégalité ci-dessus.

Q.E.D.

Remarque 4.7. Pour tout $Y \in \mathscr{L}$, on identifie $X$ et $Y \times Y^{\perp}$; on peut donc mettre tout $x \in X$ sous la forme $x=\left(y, y^{\perp}\right)$, avec $y \in Y, y^{\perp} \in Y^{\perp}$. 
Lemme 4.8. Soient $\phi \in L_{\ell o c}^{\infty}(X)$ et $\theta \in[-1,1]$ tels que $\langle.\rangle^{1+\theta}|\nabla \phi| \in \mathbb{L}^{\infty}(X)$.

(a) Si $Y \in \mathscr{L}, f \in C_{0}^{\infty}(Y)$ et $T \in \mathscr{B}\left(L^{2}(X)\right)$ est l'opérateur défini par

$$
T u(x)=\int_{Y} f(y-\eta) u\left(\eta, y^{\perp}\right) d \eta, \quad u \in L^{2}(X), \quad x=\left(y, y^{\perp}\right),
$$

alors $\langle.\rangle^{1+\theta}[T, \phi]$ (qui est bien défini sur $\left.C_{0}^{\infty}(X)\right)$ admet une unique extension en tant qu'élément de $\mathscr{B}\left(L^{2}(X)\right)$.

(b) En particulier, $\langle.\rangle^{\theta}[A, \phi] \in \mathscr{B}\left(L^{2}(X)\right)$.

Démonstration. (a) On a pour tout $u \in C_{0}^{\infty}(X)$,

$$
[T, \phi] u(x)=\int_{Y}\left[\phi\left(\eta, y^{\perp}\right)-\phi\left(y, y^{\perp}\right)\right] f(y-\eta) u\left(\eta, y^{\perp}\right) d \eta, \quad x=\left(y, y^{\perp}\right) .
$$

On note l'existence d'une constante $C_{0}>0$ telle que si $y, \eta \in Y, y-\eta \in$ $\operatorname{supp} f$ et $y^{\perp} \in Y^{\perp}$, on ait $\left|\phi\left(\eta, y^{\perp}\right)-\phi\left(y, y^{\perp}\right)\right| \leq C_{0}\langle x\rangle^{-1-\theta}, x=\left(y, y^{\perp}\right)$. On en déduit une autre constante $C>0$, telle que $\left\|\langle.\rangle^{1+\theta}[T, \phi] u\right\|_{L^{2}(X)} \leq C\|u\|_{L^{2}(X)}$ pour tout $u \in C_{0}^{\infty}(X)$.

(b) résulte de (a) et de l'expression de $A$.

Q.E.D.

Lemme 4.9. Soit $\chi \in C^{\infty}(X)$ telle que $\chi(x)=\chi(x /|x|)$ pour tout $x \in X$ avec $|x| \geq 1$. Alors pour tout $s \in \mathbb{R}$, il existe une constante $C>0$ telle que pour tout $\tau \in[-1,1]$ on ait

$$
\left\|\left[e^{\imath \tau A}, \chi\right]\right\|_{\mathscr{B}\left(\mathscr{H}^{s}(X)\right)} \leq C|\tau| .
$$

Démonstration. L'opérateur de multiplication avec $\chi$ est borné sur $\mathscr{H}^{s}(X)$ pour tout $s \in \mathbb{R}$ et d'après le lemme $4.4(\mathrm{~d}), e^{i \tau A} \in \mathscr{B}\left(\mathscr{H}^{s}(X)\right)$ pour tous $s, \tau \in \mathbb{R}$; on a de plus (4.8). Alors $\left[e^{i \tau A}, \chi\right] \in \mathscr{B}\left(\mathscr{H}^{s}(X)\right)$ et par le lemme 4.8 (b) avec $\theta=$ $0,[A, \chi] \in \mathscr{B}\left(L^{2}(X)\right)$; en fait, on vérifie facilement que $[A, \chi] \in \mathscr{B}\left(\mathscr{H}^{s}(X)\right)$ pour tout $s \in \mathbb{R}$. On peut donc utiliser la relation 6.3 .23 de [2] et écrire l'égalité

$$
\left[e^{i \tau A}, \chi\right]=i \int_{0}^{\tau} e^{i \sigma A}[\chi, A] e^{i(\tau-\sigma) A} d \sigma
$$

dans $\mathscr{B}\left(\mathscr{H}^{s}(X)\right)$, d'où l'inégalité (4.10).

Q.E.D.

Lemme 4.10. Si $Y \in \mathscr{L}$ et $Z \in \mathscr{L}_{m}(Y)$, alors $\left\langle\pi_{Y}\right\rangle^{\theta}\left[V_{Y}^{S}, A_{Z}\right] \in \mathscr{B}\left(\mathscr{H}^{1}(X)\right.$, $\left.\mathscr{H}^{-1}(X)\right)$.

Démonstration. Sur $C_{0}^{\infty}(X)$ on a l'égalité

$$
\left\langle\pi_{Y} \cdot\right\rangle^{\theta}\left[V_{Y}^{S}, A_{Z}\right]=\left\langle\pi_{Y} \cdot\right\rangle^{1+\theta} V_{Y}^{S} \cdot\left\langle\pi_{Y} \cdot\right\rangle^{-1} A_{Z}-\left\langle\pi_{Y} \cdot\right\rangle^{\theta} A_{Z}\left\langle\pi_{Y} \cdot\right\rangle^{-1-\theta}\left\langle\pi_{Y} \cdot\right\rangle^{1+\theta} V_{Y}^{S} .
$$

La propriété de l'énoncé est alors une conséquence des deux faits évidents suivants:

(a) D'après l'hypothèse III-(i) et le lemme 2.5.1 de $[1],\left\langle\pi_{Y} \cdot\right\rangle^{1+\theta} V_{Y}^{S} \in$ $\mathscr{B}\left(\mathscr{H}^{1}(X), \mathscr{H}^{-1}(X)\right)$. 
(b) Pour tous $\alpha, \beta \in \mathbb{R}$, tels que $\alpha+\beta \leq-1$ et tout $s \in \mathbb{R}$, $\left\langle\pi_{Y}\right\rangle^{\alpha} A_{Z}\left\langle\pi_{Y}\right\rangle^{\beta} \in \mathscr{B}\left(\mathscr{H}^{s}(X)\right)$.

Q.E.D.

Lemme 4.11. Si $Y \in \mathscr{L}$ et $Z \in \mathscr{L}_{m}(Y)$, alors $\left\langle\pi_{Y} \cdot\right\rangle^{\theta}\left[V_{Y}^{L}, A_{Z}\right] \in \mathscr{B}\left(\mathscr{H}^{1}(X)\right.$, $\left.\mathscr{H}^{-1}(X)\right)$.

Démonstration. Il suffit de considérer le cas $Y=X$; on note $V:=V_{X}^{L}$.

(a) Prouvons d'abord que l'on peut supposer $V \in C^{\infty}(X)$. Soit $\chi \in$ $C_{0}^{\infty}(X)$ réelle, $\chi \geq 0, \int_{X} \chi(x) d x=1$ et posons $\chi_{\varepsilon}(x):=\varepsilon^{-\operatorname{dim} X} \chi(x / \varepsilon)$ pour $x \in X$, $0<\varepsilon \leq 1$.

Si $V_{\varepsilon}:=V * \chi_{\varepsilon}$, alors $V_{\varepsilon} \in C^{\infty}(X)$ est réelle et $\lim _{\varepsilon \rightarrow 0} V_{\varepsilon}=V$ dans $\mathscr{D}^{\prime}(X)$.

Pour $u, v \in C_{0}^{\infty}(X)$ on a l'égalité

$$
\left(\langle\cdot\rangle^{\theta} V_{\varepsilon} u, v\right)=\int_{X} \chi_{\varepsilon}(z)\left(\langle\cdot\rangle^{\theta} V,\langle\cdot\rangle^{-\theta}\langle.+z\rangle^{\theta} u(.+z) \overline{v(.+z)}\right) d z,
$$

où l'on désigne par $(.,$.$) la forme sesquilinéaire sur \mathscr{D}^{\prime}(X) \times C_{0}^{\infty}(X)$, extension continue du produit scalaire sur $L^{2}(X)$. De l'hypothèse III-(iii) on déduit facilement l'existence d'une constante $C_{1}>0$ telle que l'on ait

$$
\left\|\langle\rangle^{\theta} V_{\varepsilon}\right\|_{\mathscr{B}\left(\mathscr{H}^{1}(X), \mathscr{H}^{-1}(X)\right)} \leq C_{1} \text { pour tout } \varepsilon \in(0,1]
$$

De la même façon on déduit l'existence d'une constante $C_{2}>0$ telle que l'on ait

$$
\begin{aligned}
& \left\|\langle.\rangle^{1+\theta} \xi . \nabla V_{\varepsilon}\right\|_{\mathscr{B}\left(\mathscr{H}^{1}(X), \mathscr{H}^{-1}(X)\right)} \\
& \quad \leq C_{2} \text { pour tous } \varepsilon \in(0,1] \text { et } \xi \in X,|\xi| \leq 1
\end{aligned}
$$

Supposons maintenant que l'on a prouvé le fait suivant:

$$
\langle\rangle^{\theta}\left[V_{\varepsilon}, A_{Z}\right] \in \mathscr{B}\left(\mathscr{H}^{1}(X), \mathscr{H}^{-1}(X)\right) \text { uniformément pour } \varepsilon \in(0,1] .
$$

Alors, pour tous $u, v \in C_{0}^{\infty}(X)$ on a $\left(\left[A_{Z}, V_{\varepsilon}\right] u, v\right)=\left(V_{\varepsilon} u, A_{Z} v\right)-\left(A_{Z} u, V_{\varepsilon} v\right)$, donc $\lim _{\varepsilon \rightarrow 0}\left(\left[A_{Z}, V_{\varepsilon}\right] u, v\right)=\left(\left[A_{Z}, V\right] u, v\right)$ et on aura $\langle.\rangle^{\theta}\left[V, A_{Z}\right] \in \mathscr{B}\left(\mathscr{H}^{1}(X)\right.$, $\left.\mathscr{H}^{-1}(X)\right)$.

(b) Prouvons maintenant (4.13). En tenant compte de la forme de $A_{Z}$ il suffit de prouver que

$$
\langle.\rangle^{1+\theta}\left[T, V_{\varepsilon}\right] \in \mathscr{B}\left(\mathscr{H}^{1}(X), \mathscr{H}^{-1}(X)\right)
$$

uniformément pour $\varepsilon \in(0,1]$, où $T: C_{0}^{\infty}(X) \rightarrow C_{0}^{\infty}(X)$ est un opérateur de la forme

$$
T u(x)=\int_{Z} f(z-\zeta) u\left(\zeta, z^{\perp}\right) d \zeta, \quad u \in C_{0}^{\infty}(X)
$$


avec $f \in C_{0}^{\infty}(Z)$ et $x=\left(z, z^{\perp}\right), z \in Z, z^{\perp} \in Z^{\perp}$. Un calcul direct du commutateur $\left[T, V_{\varepsilon}\right]$, l'égalité $V_{\varepsilon}\left(z, z^{\perp}\right)-V_{\varepsilon}\left(\zeta, z^{\perp}\right)=(z-\zeta) \cdot \int_{0}^{1}\left(\nabla_{\zeta} V_{\varepsilon}\right)\left(t z+(1-t) \zeta, z^{\perp}\right) d t$ et deux changements de variables prouvent que l'on a, pour tous $u, v \in C_{0}^{\infty}(X)$,

$$
\begin{aligned}
& \left(\langle.\rangle^{1+\theta}\left[T, V_{\varepsilon}\right] u, v\right) \\
& \quad=\int_{0}^{1}\left\{\int_{Z}\langle z\rangle f\langle z\rangle\left[\int_{X}\langle\tilde{\zeta}\rangle^{1+\theta} \frac{z}{\langle z\rangle} \cdot \nabla_{\zeta} V_{\varepsilon}(\tilde{\zeta}) \tilde{u}(t, z, \tilde{\zeta}) \overline{\tilde{v}(t, z, \tilde{\zeta})} d \tilde{\zeta}\right] d z\right\} d t
\end{aligned}
$$

où $\tilde{\zeta}=\left(\zeta, z^{\perp}\right)$ avec $\zeta \in Z, z^{\perp} \in Z^{\perp}, \tilde{u}(t, z, \tilde{\zeta}):=u\left(\zeta-t z, z^{\perp}\right), \tilde{v}(t, z, \tilde{\zeta}):=\langle\tilde{\zeta}\rangle^{-1-\theta}$. $w\left(\zeta+(1-t) z, z^{\perp}\right), \quad w(x):=\langle x\rangle^{1+\theta} v(x)$. En utilisant (4.12), on en déduit facilement (4.14). Q.E.D.

Lemme 4.12. Soient $Y \in \mathscr{L}, Z \in \mathscr{L}_{m}(Y), \chi \in C^{\infty}(X)$ telle que $\chi\left(\frac{x}{|x|}\right)$ $=\chi(x)$ pour tout $x \in X,|x| \geq 1$ et $\Phi^{Y} \in L_{\text {loc }}^{1}(Y)$ telle que $\langle.\rangle \Phi^{Y} \in \mathscr{B}\left(\mathscr{H}^{1}(Y)\right.$, $\left.\mathscr{H}^{-1}(Y)\right) . \quad$ Si $\Phi_{Y}:=\Phi^{Y} \otimes 1_{Y^{\perp}} \in L_{\ell o c}^{1}(X)$, alors

$\langle\rangle.\left[\chi,\left[A_{Z}, \Phi_{Y}\right]\right] \in \mathscr{B}\left(\mathscr{H}^{1}(X), \mathscr{H}^{-1}(X)\right)$.

Démonstration. Le commutateur ci-dessus est bien défini sur $C_{0}^{\infty}(X)$ et on voit qu'il suffit de prouver que $\langle\rangle.\left[\chi, A_{Z} \Phi_{Y}\right] \in \mathscr{B}\left(\mathscr{H}^{1}(X), \mathscr{H}^{-1}(X)\right)$.

En tenant compte de l'expression de $A_{Z}$, il suffit en fait de supposer $Y=X$ et de prouver que si $\Phi \in L_{\ell o c}^{1}(X), \Phi \in \mathscr{B}\left(\mathscr{H}^{1}(X), \mathscr{H}^{-1}(X)\right)$ et $T: \mathscr{D}^{\prime}(X) \rightarrow$ $\mathscr{D}^{\prime}(X)$ est un opérateur de la forme (4.15), alors $\langle\rangle.[\chi, T \Phi] \in \mathscr{B}\left(\mathscr{H}^{1}(X)\right.$, $\left.\mathscr{H}^{-1}(X)\right)$.

Or, un calcul direct du commutateur, l'égalité $\chi\left(z, z^{\perp}\right)-\chi\left(\zeta, z^{\perp}\right)=(z-\zeta)$. $\int_{0}^{1}\left(\nabla_{Z} \chi\right)\left(t z+(1-t) \zeta, z^{\perp}\right) d t$ et un changement de variables, prouvent que si l'on désigne encore par $(.,$.$) l'extension sesquilinéaire à \mathscr{D}^{\prime}(X) \times C_{0}^{\infty}(X)$ du produit scalaire de $L^{2}(X)$ on a, pour tous $u, v \in C_{0}^{\infty}(X)$,

$$
\begin{aligned}
(\langle.\rangle[\chi, T \Phi] u, v)= & \int_{0}^{1}\left\{\int _ { Z } \langle z \rangle f ( z ) \left[\int_{X} \Phi\left(\zeta, z^{\perp}\right)\left(1+|\zeta+z|^{2}+\left|z^{\perp}\right|^{2}\right)^{1 / 2} \frac{z}{\langle z\rangle}\right.\right. \\
& \left.\left.\left(\nabla_{z} \chi\right)\left(\zeta+t z, z^{\perp}\right) u\left(\zeta, z^{\perp}\right) \overline{v\left(z, z^{\perp}\right)} d \zeta d z^{\perp}\right] d z\right\} d t
\end{aligned}
$$

et donc, en utilisant la propriété d'homogénéité de $\chi$ et le fait que $\operatorname{supp} f$ est un compact, on en déduit l'existence d'une constante $C>0$ telle que

$$
|(\langle.\rangle[\chi, T \Phi] u, v)| \leq C\|u\|_{\mathscr{H}^{1}(X)}\|v\|_{\mathscr{H}^{1}(X)}, \quad u, v \in C_{0}^{\infty}(X)
$$

Q.E.D.

Lemme 4.13. Soient $Y \in \mathscr{L}, Z \in \mathscr{L}_{m}(Y), \chi \in C^{\infty}(X)$ telle que $\chi\left(\frac{x}{|x|}\right)=$ $\chi(x)$ pour tout $x \in X,|x| \geq 1$ et $\Phi^{Y} \in L_{\ell o c}^{1}(Y), \Phi^{Y} \in \mathscr{B}\left(\mathscr{H}^{1}(Y), \mathscr{H}^{-1}(Y)\right)$, tel 
que pour tout $\eta \in Y,\langle.\rangle \eta \cdot \nabla \Phi^{Y} \in \mathscr{B}\left(\mathscr{H}^{1}(Y), \mathscr{H}^{-1}(Y)\right)$ uniformément pour $|\eta| \leq 1$.

$$
\text { Si } \Phi_{Y}:=\Phi^{Y} \otimes 1_{Y^{\perp}} \text {, alors }\langle.\rangle\left[\chi,\left[A_{Z}, \Phi_{Y]}\right] \in \mathscr{B}\left(\mathscr{H}^{1}(X), \mathscr{H}^{-1}(X)\right)\right. \text {. }
$$

Démonstration. Le commutateur de l'énoncé étant bien défini sur $C_{0}^{\infty}(X)$, on voit (de la même manière que pour la preuve du lemme 4.11) que l'on peut supposer $Y=X, \Phi:=\Phi_{X}=\Phi^{X} \in C^{\infty}(X), \Phi \in \mathscr{B}\left(\mathscr{H}^{1}(X), \mathscr{H}^{-1}(X)\right),\langle.\rangle \xi . \nabla \Phi \in$ $\mathscr{B}\left(\mathscr{H}^{1}(X), \mathscr{H}^{-1}(X)\right)$ uniformément pour $\xi \in X,|\xi| \leq 1$ et prouver que si $T: \mathscr{D}^{\prime}(X) \rightarrow \mathscr{D}^{\prime}(X)$ est de la forme (4.15), alors $\left.\left\langle\pi_{Z} \cdot\right\rangle \cdot\right\rangle\left[\chi,\left[T, \Phi_{]}\right] \in\right.$ $\mathscr{B}\left(\mathscr{H}^{1}(X), \mathscr{H}^{-1}(X)\right)$. Avec les notations de (4.16) (sauf pour $w(x):=$ $\langle x\rangle\left\langle\pi_{Z} z\right\rangle v(x)$ et $\tilde{v}(t, z, \tilde{\zeta}):=\langle\tilde{\zeta}\rangle^{-1} w\left(\zeta+(1-t) z, z^{\perp}\right)$ et en utilisant l'égalité $\chi\left(z, z^{\perp}\right)-\chi\left(\zeta, z^{\perp}\right)=(z-\zeta) \cdot \int_{0}^{1}\left(\nabla_{z} \chi\right)\left(t z+(1-t) \zeta, z^{\perp}\right) d t$, on obtient que pour tous $u, v \in C_{0}^{\infty}(X)$ on a

$$
\begin{aligned}
\left(\left\langle\pi_{Z} \cdot\right\rangle\langle\rangle[\chi,[T, \Phi]] u, v\right)= & \int_{0}^{1} \int_{0}^{1}\left\{\int _ { Z } \langle z \rangle ^ { 2 } f ( z ) \left[\int_{X}\langle\tilde{\zeta}\rangle \frac{z}{\langle z\rangle} . \nabla \zeta \Phi(\tilde{\zeta})\right.\right. \\
& \left.\left.\frac{z}{\langle z\rangle} \cdot\left(\nabla_{z} \chi\right)\left(\zeta+(s-t) z, z^{\perp}\right) \tilde{u}(t, z, \tilde{\zeta}) \overline{\tilde{v}}(t, z, \tilde{\zeta}) d \tilde{\zeta}\right] d z\right\} d s d t .
\end{aligned}
$$

En utilisant l'homogénéité de $\chi$, ainsi que le fait que $\operatorname{supp} f$ est un compact, on obtient l'existence d'une constante $C>0$ telle que

$$
\left|\left(\left\langle\pi_{Z}\right\rangle\langle.\rangle[\chi,[T, \Phi]] u, v\right)\right| \leq C\|u\|_{\mathscr{H}^{1}(X)}\|v\|_{\mathscr{H}^{1}(X)}, \quad u, v \in C_{0}^{\infty}(X) .
$$

Q.E.D.

\section{§5. Régularité de $H$ par Rapport à l'Opérateur Conjugué}

Sous les hypothèses I-IV, l'opérateur conjugué $A$ a été défini dans la section précédente. Si $H=H(\rho, V)$ est défini par (1.2) et (1.3), on peut considérer le commutateur $[H, A]$ défini en tant qu'application linéaire de $C_{0}^{\infty}(X)$ dans $\mathscr{D}^{\prime}(X)$ par:

$$
\begin{aligned}
& ([H, A] u, v)=\overline{(H v, A u)}-(H u, A v) \\
& \quad=\int_{X} \rho(\nabla A u \cdot \overline{\nabla v}-\nabla u \cdot \overline{\nabla A v}) d x+\int_{X} V[(A u) \bar{v}-\overline{u(A v)}] d x, \quad u, v \in C_{0}^{\infty}(X),
\end{aligned}
$$

où l'on désigne, comme d'habitude, par $(.,$.$) l'extension en tant que forme$ sesquilinéaire sur $\mathscr{D}^{\prime}(X) \times C_{0}^{\infty}(X)$ du produit scalaire sur $L^{2}(X)$.

La preuve de la régularité (au sens du §2) de $H$ par rapport à $A$ (et aussi bien celle de l'inégalité de Mourre) repose sur un raisonnement par récurrence sur $Y \in \mathscr{L}$ qui nécessite une relation qui lie $[H, A]$ aux commutateurs $\left[H_{Y}, A\right]$ pour $Y \in \mathscr{L}_{1}$. Dans ce but on prouve d'abord deux lemmes. 
Lemme 5.1. Pour tous $u, v \in C_{0}^{\infty}(X)$ on a l'égalité

$$
\begin{aligned}
([H, A] u, v)= & \int_{X} \rho(F u \cdot \overline{\nabla v}-\nabla u \cdot \overline{F v}) d x \\
& +\sum_{Z \in \mathscr{L}} \int_{X} \delta_{Z}\left[A_{Z}(\nabla u) \cdot \nabla \bar{v}-\nabla u \cdot \overline{A_{Z}(\nabla v)} d x\right. \\
& +\sum_{Z \in \mathscr{L}} \int_{X} V_{Z}\left[\left(A_{Z} u\right) \bar{v}-\overline{u\left(A_{Z} v\right)}\right] d x
\end{aligned}
$$

Démonstration. Il suffit d'utiliser (5.1), le lemme 4.4 (b), les égalités $\rho=\rho^{0}+\sum_{Z \in \mathscr{L}} \delta_{Z}, V=\sum_{Z \in \mathscr{L}} V_{Z}$ et le fait que l'on a pour tout $Z \in \mathscr{L}$,

$$
\int_{Z^{\perp}}\left[A_{Z^{\perp}}(\nabla u) \cdot \nabla \bar{v}-\nabla u \cdot \overline{A_{Z^{\perp}}(\nabla v)}\right] d z=0, \quad \int_{Z^{\perp}}\left[\left(A_{Z^{\perp}} u\right) \bar{v}-u \overline{\left(A_{Z^{\perp}} v\right)}\right] d z=0
$$

car $A^{Z^{\perp}}$ est auto-adjoint sur $L^{2}\left(Z^{\perp}\right)$ et $C_{0}^{\infty}\left(Z^{\perp}\right) \subset D\left(A^{Z^{\perp}}\right)$.

Q.E.D.

Lemme 5.2. Si $\Phi \in L_{\ell o c}^{\infty}(X)$ et $u, v \in C_{0}^{\infty}(X)$, alors pour tout $Z \in \mathscr{L}$ on a l'égalité

$$
\int_{X} \Phi\left[A_{Z}(\nabla u) \cdot \nabla \bar{v}-\nabla u \cdot \overline{A_{Z}(\nabla v)}\right] d x=\int_{X}\left[\Phi, A_{Z}\right](\nabla u) \cdot \nabla \bar{v} d x
$$

où le commutateur $\left[\Phi, A_{Z}\right]$ est défini de façon naturelle en tant qu'opérateur continu sur $L_{\text {comp }}^{2}(X ; X \otimes \mathbb{C})$.

Démonstration. Il suffit d'utiliser les faits suivants: $A_{Z}$ est auto-adjoint sur $L^{2}(X)$ et pour tout $f \in C_{0}^{\infty}(X), \Phi f \in D\left(A_{Z}\right)$.

Q.E.D.

Proposition 5.3. Soient $\left\{\chi_{Y}\right\}_{Y \in \mathscr{L}_{1}}$ la partition de l'unité de la définition 3.4 et $\theta \in(0,1]$ des hypothèses II et III. Il existe alors un opérateur $S \in \mathscr{B}\left(\mathscr{H}^{1}(X)\right.$, $\left.\mathscr{H}^{-1}(X)\right)$ tel que $\left\langle.{ }^{\theta} S \in \mathscr{B}\left(\mathscr{H}^{1}(X), \mathscr{H}^{-1}(X)\right.\right.$ et que l'on ait sur $C_{0}^{\infty}(X)$ :

$$
[H, A]=\sum_{Y \in \mathscr{L}_{1}} \chi_{Y}\left[H_{Y}, A\right] \chi_{Y}+S
$$

Démonstration. On utilise l'identité $\sum_{Y \in \mathscr{L}_{1}} \chi_{Y}^{2}=1$ sur $X$ sous chacune des intégrales de (5.2). Au bout de quelques opérations de commutation des opérateurs de multiplication par $\chi_{Y}$ avec $F, \nabla, A_{Z}$, en séparant les commutateurs $\left[H_{Y}, A\right]\left(Y \in \mathscr{L}_{1}\right)$ définis par la même formule (5.2) avec $\rho_{Y}$ à la place de $\rho$ et les deux sommes sur $Z \in \mathscr{L}(Y)$, on voit que l'opérateur $S: C_{0}^{\infty}(X) \rightarrow$ $\mathscr{D}^{\prime}(X)$, qui vérifie $(5.5)$, est de la forme 


$$
\begin{aligned}
&(S u, v):= \sum_{Y \in \mathscr{L}_{1}} \sum_{J=1}^{3} E_{Y}^{(J)}(u, v)+\sum_{Y \in \mathscr{L}_{1}} \sum_{Z \in \mathscr{L}(Y)} \sum_{J=1}^{3} E_{Y, Z}^{(J)}(u, v) \\
&+\sum_{Y \in \mathscr{L}_{1}} \sum_{Z \in \mathscr{L} \backslash \mathscr{L}(Y)} \sum_{j=4}^{5} E_{Y, Z}^{(J)}(u, v), \\
& E_{Y}^{(J)}(u, v):= I_{Y}^{(J)}(u, v)-\overline{I_{Y}^{(j)}(v, u)}, \quad E_{Y, Z}^{(J)}(u, v)=I_{Y, Z}^{(J)}(u, v)-\overline{I_{Y, Z}^{(J)}(v, u)} \\
& u, v \in C_{0}^{\infty}(X),
\end{aligned}
$$

où les expressions $I_{Y}^{(J)}, I_{Y, Z}^{(J)}$ seront explicitées ci-dessous. Il suffit de prouver qu'il existe une constante $C>0$ telle que pour tous $f, g \in C_{0}^{\infty}(X)$ on ait

$$
\left|\left(S f,\langle\cdot\rangle^{\theta} g\right)\right| \leq C\|f\|_{\mathscr{H}^{1}(X)}\|g\|_{\mathscr{H}^{1}(X)} .
$$

On va utiliser la notation suivante: si $E_{1}(f, g)$ et $E_{2}(f, g)$ sont deux expressions dépendant de $f, g \in C_{0}^{\infty}(X)$, on écrira $E_{1} \sim E_{2}$ si $\left|E_{1}-E_{2}\right|$ peut être estimé par le terme du côté droit de (5.7).

$$
I_{Y}^{(1)}(u, v)=\int_{X} \chi_{Y}^{2}\left(\rho-\rho_{Y}\right)(F u) . \nabla \bar{v} d x, \quad Y \in \mathscr{L}_{1} .
$$

D'après l'hypothèse II et la remarque 3.5 (b), il existe une constante $C>0$ telle que $\left|\chi_{Y}^{2}\left(\rho-\rho_{Y}\right)(x)\right| \leq C\langle x\rangle^{-\theta}, x \in X$, d'où l'on déduit facilement que $I_{Y}^{(1)}\left(f,\langle.\rangle^{\theta} g\right) \sim 0$. Si on regarde l'expression de $F$ et le lemme 4.8 (a), on voit que $I_{Y}^{(1)}\left(\langle\cdot\rangle^{\theta} f, g\right) \sim 0$, donc $E_{Y}^{(1)}\left(f,\langle.\rangle^{\theta} g\right) \sim 0$.

ii)

$$
I_{Y}^{(2)}(u, v)=\int_{X} \rho_{Y} \chi_{Y}(F u) \cdot \overline{\left(\left[\chi_{Y}, \nabla\right] v\right)} d x, \quad Y \in \mathscr{L}_{1} .
$$

D'après la propriété d'homogénéité de $\chi_{Y}$, il existe une constante $C>0$ telle que $\left|\nabla \chi_{Y}(x)\right| \leq C\langle x\rangle^{-1}$ et puisque $\rho_{Y} \in L^{\infty}(X)$, nous aurons $I_{Y}^{(2)}\left(f,\langle\cdot\rangle^{\theta} g\right) \sim 0$.

En utilisant encore une fois le lemme 4.8(a), on obtient aussi $I_{Y}^{(2)}\left(\langle.\rangle^{0} f, g\right)$ $\sim 0$ et $E_{Y}^{(2)}\left(f,\langle.\rangle^{\theta} g\right) \sim 0$.

iii)

$$
I_{Y}^{(3)}(u, v)=\int_{X} \rho_{Y}\left(\left[\chi_{Y}, F\right] u\right) \cdot \overline{\left(\nabla\left(\chi_{Y} v\right)\right)} d x, \quad Y \in \mathscr{L}_{1} .
$$

D'apprès le lemme 4.8 (a), $\langle\rangle.\left[\chi_{Y}, F\right] \in \mathscr{B}\left(L^{2}(X), L^{2}(X, X \otimes \mathbb{C})\right)$ et alors $I_{Y}^{(3)}\left(f,\langle.\rangle^{\theta} g\right) \sim 0$. Le même lemme 4.8 (a) et un argument de dualité montre que l'on a aussi $\left[\chi_{Y}, F\right]\langle.\rangle \in \mathscr{B}\left(L^{2}(X), L^{2}(X, X \otimes \mathbb{C})\right)$, donc $I_{Y}^{(3)}\left(\langle\cdot\rangle^{0} f, g\right) \sim 0$ et aussi $E_{Y}^{(3)}\left(f,\langle.\rangle^{\theta} g\right) \sim 0$.

iv) $\quad I_{Y, Z}^{(1)}(u, v)=\int_{X} \delta_{Z} \chi_{Y}\left(A_{Z}(\nabla u)\right) \cdot \overline{\left(\left[\chi_{Y}, \nabla\right] v\right)} d x, \quad Y \in \mathscr{L}_{1}, \quad Z \in \mathscr{L}(Y)$. 
Pour estimer $I_{Y, Z}^{(1)}\left(f,\langle\rangle^{\theta} g\right)$, on utilise la propriété du $\left|\nabla \chi_{Y}\right|$ de (ii), l'hypothèse II et l'expression de $A_{Z}$; on arrive à une expression de la forme

$$
\int_{X}\left\langle\pi_{Z} x\right\rangle^{1-\theta}\langle x\rangle^{\theta-1}(M f) \overline{(N g)} d x
$$

où $M \in \mathscr{B}\left(\mathscr{H}^{1}(X), L^{2}(X)\right), \quad N \in \mathscr{B}\left(L^{2}(X)\right)$, donc $I_{Y, Z}^{(1)}\left(f,\langle\cdot\rangle^{\theta} g\right) \sim 0$. Si on utilise de plus de lemme 4.8(a), on obtient de la même manière que $I_{Y, Z}^{(1)}\left(\langle.\rangle^{\theta} f, g\right) \sim 0 . \quad$ Donc $E_{Y, Z}^{(1)}\left(f,\langle.\rangle^{\theta} g\right) \sim 0$.

v) $\quad I_{Y, Z}^{(2)}(u, v)=\int_{X} \delta_{Z}\left(\left[\chi_{Y}, A_{Z} \nabla\right] u\right) \cdot \overline{\nabla\left(\chi_{Y} v\right)} d x, \quad Y \in \mathscr{L}_{1}, \quad Z \in \mathscr{L}(Y)$.

En utilisant l'identité $\left[\chi_{Y}, A_{Z} \nabla\right]=\left[\chi_{Y}, A_{Z}\right] \nabla+A_{Z}\left[\chi_{Y}, \nabla\right]$ sur $C_{0}^{\infty}(X)$, on prouve que $I_{Y, Z}^{(2)}\left(f,\langle\rangle^{\theta} g\right) \sim 0$ de la même manière qu'au point (iv). Si on tient compte du fait que dans l'énoncé du lemme 4.8 (a) on peut intervertir les deux facteurs $\langle.\rangle^{1+\theta}$ et $[T, \Phi]$ (raisonnement direct, ou par dualité) on s'aperçoit que l'on a aussi $I_{Y, Z}^{(2)}\left(\langle\cdot\rangle^{\theta} f, g\right) \sim 0$ et alors $E_{Y, Z}^{(2)}\left(f,\langle\cdot\rangle^{\theta} g\right) \sim 0$.

$$
E_{Y, Z}^{(3)}(u, v)=\left(\left[\chi_{Y},\left[V_{Z}, A_{Z}\right]\right] u, \chi_{Y} v\right), \quad Y \in \mathscr{L}_{1}, Z \in \mathscr{L}(Y) .
$$

Si on utilise l'hypothèse III et les lemmes 4.12 et 4.13 , on obtient directement que $E_{Y, Z}^{(3)}\left(f,\langle\cdot\rangle^{\theta} g\right) \sim 0$.

vii) $\quad I_{Y, Z}^{(4)}(u, v)=\int_{X} \chi_{Y}^{2} \delta_{Z}\left(A_{Z}(\nabla u)\right) \cdot \overline{(\nabla v)} d x, \quad Y \in \mathscr{L}_{1}, \quad Z \in \mathscr{L} \backslash \mathscr{L}(Y)$.

D'après le lemme 5.2,

$$
E_{Y, Z}^{(4)}(u, v):=I_{Y, Z}^{(4)}(u, v)-\overline{I_{Y, Z}^{(4)}(u, v)}=\int_{X}\left\{\left[\chi_{Y}^{2} \delta_{Z}, A_{Z}\right](\nabla u)\right\} \cdot \overline{\nabla v} d x .
$$

En tenant compte de l'hypothèse II et des propriétés du support de $\chi_{Y}$ (voir la remarque 3.5 (b)), il existe une constante $C>0$ telle que l'on ait $\left|\left(\chi_{Y}^{2} \delta_{Z}^{S}\right)(x)\right| \leq C\langle x\rangle^{-1-\theta}, \quad\left|\left(\chi_{Y}^{2} \delta_{Z}^{L}\right)(x)\right| \leq C\langle x\rangle^{-\theta} \quad$ et $\quad\left|\nabla\left(\chi_{Y}^{2} \delta_{Z}^{L}\right)(x)\right| \leq C\langle x\rangle^{-1-\theta}$ pour tout $x \in X$. Alors $\langle.\rangle^{\theta}\left[\chi_{Y}^{2} \delta_{Z}^{S}, A_{Z}\right]=\langle.\rangle^{\theta} \chi_{Y}^{2} \delta_{Z}^{S} A_{Z}-\langle.\rangle^{\theta} A_{Z} \chi_{Y}^{2} \delta_{Z}^{S} \in$ $\mathscr{B}\left(L^{2}(X)\right)$ et d'après le lemme 4.8 (b), 〈. $\rangle^{\theta}\left[\chi_{Y}^{2} \delta_{Z}^{L}, A_{Z}\right] \in \mathscr{B}\left(L^{2}(X)\right)$, donc $\langle.\rangle^{\theta}\left[\chi_{Y}^{2} \delta_{Z}, A_{Z}\right]$ appartient à $\mathscr{B}\left(L^{2}(X)\right)$. Il en résulte que $E_{Y, Z}^{(4)}\left(f,\langle.\rangle^{\theta} g\right) \sim 0$.

$$
E_{Y, Z}^{(5)}(u, v)=\left(\chi_{Y}^{2}\left[V_{Z}, A_{Z}\right] u, v\right), \quad Y \in \mathscr{L}_{1}, \quad Z \in \mathscr{L} \backslash \mathscr{L}(Y) .
$$

D'après les lemmes $4.10,4.11$ et les propriétés du support de $\chi_{Y}$, on a $\langle.\rangle^{\theta} \chi_{Y}^{2}\left[V_{Z}, A_{Z}\right] \in \mathscr{B}\left(\mathscr{H}^{1}(X), \mathscr{H}^{-1}(X)\right)$, donc $E_{Y, Z}^{(5)}\left(f,\langle.\rangle^{\theta} g\right) \sim 0$.

Q.E.D.

On peut maintenant étudier la régularité de $H$ par rapport à $A$.

Proposition 5.4 Sous les hypothèses I-IV, $H$ appartient à $C^{1+\theta}(A$; $\left.\mathscr{H}^{1}(X), \mathscr{H}^{-1}(X)\right)$. 
Démonstration. On procède par récurrence suivant le sous-espace $Y \in \mathscr{L}$. Plus exactement:

a) Si $Y=\{0\}$, par convention $L^{2}(Y)=\mathbb{C}, H^{Y}=V^{0}, A^{Y}=0$ et il n'y a rien à prouver.

b) Supposons maintenant que pour un $Z \in \mathscr{L}$, on a prouvé que $H^{Y} \in$ $C^{1+\theta}\left(A^{Y} ; \mathscr{H}^{1}(Y), \mathscr{H}^{-1}(Y)\right)$ pour tous $Y \in \mathscr{L}(Z), Y \neq Z$. Il faudra vérifier que $H^{Z} \in C^{1+\theta}\left(A^{Z} ; \mathscr{H}^{1}(Z), \mathscr{H}^{-1}(Z)\right)$. On peut très bien admettre que $Z=X$.

c) Démontrons d'abord que si $Y \in \mathscr{L}_{1}$ et $H^{Y} \in C^{1+\theta}\left(A^{Y} ; \mathscr{H}^{1}(Y)\right.$, $\left.\mathscr{H}^{-1}(Y)\right)$ alors $H_{Y} \in C^{1+\theta}\left(A ; \mathscr{H}^{1}(X), \mathscr{H}^{-1}(X)\right)$. Remarquons d'abord que si l'on compare les expressions de $\left[H_{Y}, A\right]$ et $\left[H^{Y}, A^{Y}\right]$ données par la formule (5.2) on obtient que pour tous $u, v \in C_{0}^{\infty}(X)$, on a

$$
\begin{aligned}
\left(\left[H_{Y}, A\right] u, v\right)= & \left(\left\{\left[H^{Y}, A^{Y}\right] \otimes 1_{Y^{\perp}}\right\} u, v\right) \\
& +\int_{X} \rho_{Y}\left\{\left(F_{Y^{\perp}} u\right) \cdot \overline{\left(\nabla_{Y^{\perp}} v\right)}-\left(\nabla_{Y^{\perp}} u\right) \cdot \overline{\left(F_{Y^{\perp}} v\right)}\right\} d x \\
& +\sum_{Z \in \mathscr{L}(Y)} \int_{X} \delta_{Z}\left\{A_{Z}\left(\nabla_{Y^{\perp}} u\right) \cdot \overline{\left(\nabla_{Y^{\perp}} v\right)}-\left(\nabla_{Y^{\perp}} u\right) \cdot \overline{A_{Z}\left(\nabla_{Y^{\perp}}\right)}\right\} d x
\end{aligned}
$$

où $F_{Y^{\perp}}:=1_{Y} \otimes F^{Y^{\perp}}: \mathscr{D}^{\prime}(X) \rightarrow \mathscr{D}^{\prime}(X ; X \otimes \mathbb{C})$ et $F^{Y^{\perp}}: \mathscr{D}^{\prime}\left(Y^{\perp}\right) \rightarrow \mathscr{D}^{\prime}\left(Y^{\perp} ; Y^{\perp} \otimes \mathbb{C}\right)$ est défini à partir de la famille $\mathscr{L}_{m}\left(Y^{\perp}\right)$ de la même façon que $F$ (voir le lemme 4.4 (b)). Il en résulte que sur $C_{0}^{\infty}(X)$ on a la relation

$$
\begin{aligned}
{\left[H_{Y}, A\right]=} & {\left[H^{Y}, A^{Y}\right] \otimes 1_{Y^{\perp}}+2 \rho^{Y} \otimes \nabla_{Y^{\perp}}^{*} F^{Y^{\perp}} } \\
& +\sum_{Z \in \mathscr{L}(Y)}\left[\delta^{Z}, A^{Z}\right] \otimes 1_{Y \ominus Z} \otimes \Delta_{Y^{\perp}} .
\end{aligned}
$$

En utilisant l'expression directe du commutateur $\left[\delta^{Z, S}, A^{Z}\right]$, ainsi que le lemme 4.8 (b) pour estimer $\left[\delta^{Z, L}, A^{Z}\right]$, on obtient que

$$
\langle\rangle^{\theta}\left[\delta^{Z}, A^{Z_{j}} \in \mathscr{B}\left(L^{2}(Z)\right)\right. \text {. }
$$

D'autre part, $\Delta_{Y^{\perp}} \in \mathscr{B}\left(\mathscr{H}^{1}\left(Y^{\perp}\right), \mathscr{H}^{-1}\left(Y^{\perp}\right)\right), \quad \rho^{Y} \in \mathscr{B}\left(L^{2}(Y)\right), \quad \nabla_{Y^{\perp}}^{*} F^{Y^{\perp}} \in$ $\mathscr{B}\left(L^{2}\left(Y^{\perp}\right)\right)$ et, par hypothèse, $\left[H^{Y}, A^{Y}\right] \in \mathscr{B}\left(\mathscr{H}^{1}(Y), \mathscr{H}^{-1}(Y)\right)$.

Il en résulte que $\left[H_{Y}, A\right] \in \mathscr{B}\left(\mathscr{H}^{1}(X), \mathscr{H}^{-1}(X)\right)$, donc $H_{Y} \in C^{1}\left(A ; \mathscr{H}^{1}(X)\right.$, $\left.\mathscr{H}^{-1}(X)\right)$.

On a en fait, d'après l'hypothèse d'induction, que $\left[H^{Y}, A^{Y}\right] \in$ $C^{\theta}\left(A ; \mathscr{H}^{1}(Y), \mathscr{H}^{-1}(Y)\right)$, donc, en utilisant le lemme 4.6, $\left[H^{Y}, A^{Y}\right] \otimes 1_{Y^{\perp}} \in$ $C^{\theta}\left(A ; \mathscr{H}^{1}(Y), \mathscr{H}^{-1}(Y)\right)$.

D'autre part, d'après (5.9), $\left[\rho^{Y}, A^{Y}\right]=\sum_{Z \in \mathscr{L}(Y)}\left[\delta^{Z}, A^{Z}\right] \otimes 1_{Y \ominus Z} \in \mathscr{B}\left(L^{2}(Y)\right)$, donc $\rho^{Y} \in C^{1}\left(A^{Y} ; L^{2}(Y)\right)$. De plus, on a

$$
\begin{aligned}
& \nabla_{Y^{\perp}}^{*} F^{Y^{\perp}}=\sum_{Z \in \mathscr{L}_{m}\left(Y^{\perp}\right)} T_{Z}, \\
& T_{Z} u\left(y^{\perp}\right)=\int_{Z} f_{Z}\left(z^{\prime}-\zeta\right) u\left(\zeta, z^{\prime \prime}\right) d \zeta, \quad u \in \mathscr{C}_{0}^{\infty}\left(Y^{\perp}\right),
\end{aligned}
$$


où $f_{Z} \in C_{0}^{\infty}(Z), y^{\perp}=\left(z^{\prime}, z^{\prime \prime}\right), z^{\prime} \in Z, z^{\prime \prime} \in Y^{\perp} \ominus Z$. Un calcul direct (ou bien une transformée de Fourier) montre que $\left[\nabla_{Y^{\perp}}^{*} F^{Y^{\perp}}, A^{Y^{\perp}}\right]$ est de la forme (5.10), donc ce commutateur est un opérateur borné sur $L^{2}\left(Y^{\perp}\right)$ et alors $\nabla_{Y^{\perp}}^{*} F^{Y^{\perp}} \in$ $C^{1}\left(A^{Y^{\perp}} ; L^{2}\left(Y^{\perp}\right)\right)$.

On en déduit du lemme 4.6 que $\rho^{Y} \otimes \nabla_{Y^{\perp}}^{*} F^{Y^{\perp}} \in C^{1}\left(A ; L^{2}(X)\right)$.

Finalement, en utilisant (5.9) et le lemme 4.5, on aura $\left[\delta^{Z}, A^{Z}\right] \in$ $C^{\theta}\left(A^{Z} ; L^{2}(Z)\right)$. D'autre part, d'après la formule $(5.8),\left[\Delta_{Y^{\perp}}, A^{Y^{\perp}}\right]=2 \nabla_{Y^{\perp}}^{*} F^{Y^{\perp}}$ $\in \mathscr{B}\left(L^{2}\left(Y^{\perp}\right)\right)$, donc $\Delta_{Y^{\perp}}$ appartient à $C^{1}\left(A^{Y^{\perp}} ; \mathscr{H}^{1}\left(Y^{\perp}\right), \mathscr{H}^{-1}\left(Y^{\perp}\right)\right)$.

Il résulte du lemme 4.6 que $\left[\delta^{Z}, A^{Z}\right] \otimes 1_{Y \ominus Z} \otimes \Delta_{Y^{\perp}} \in C^{\theta}\left(A ; \mathscr{H}^{1}(X)\right.$, $\left.\mathscr{H}^{-1}(X)\right)$.

En conclusion, $\left[H_{Y}, A\right] \in C^{\theta}\left(A ; \mathscr{H}^{1}(X), \mathscr{H}^{-1}(X)\right)$, donc $H_{Y} \in C^{1+\theta}(A ;$ $\left.\mathscr{H}^{1}(X), \mathscr{H}^{-1}(X)\right)$.

(d) Pour vérifier que $H \in C^{1+\theta}\left(A ; \mathscr{H}^{1}(X), \mathscr{H}^{-1}(X)\right)$, on utilise maintenant la relation (5.5). On a évidemment $[H, A] \in \mathscr{B}\left(\mathscr{H}^{1}(X), \mathscr{H}^{-1}(X)\right)$ et donc $H \in$ $C^{1}\left(A ; \mathscr{H}^{1}(X), \mathscr{H}^{-1}(X)\right)$. D'après le lemme $4.5, S \in C^{\theta}\left(A ; \mathscr{H}^{1}(X), \mathscr{H}^{-1}(X)\right)$ et par (c), $\left[H_{Y}, A\right] \in C^{\theta}\left(A ; \mathscr{H}^{1}(X), \mathscr{H}^{-1}(X)\right)$ et alors en utilisant le lemme 4.9 on aura $\chi_{Y}\left[H_{Y}, A\right] \chi_{Y} \in C^{\theta}\left(A ; \mathscr{H}^{1}(X), \mathscr{H}^{-1}(X)\right)$. Par conséquent $[H, A] \in$ $C^{\theta}\left(A ; \mathscr{H}^{1}(X), \mathscr{H}^{-1}(X)\right)$ et donc $H \in C^{1+\theta}\left(A ; \mathscr{H}^{1}(X), \mathscr{H}^{-1}(X)\right)$.

Q.E.D.

En utilisant certains arguments de la démonstration ci-dessus, on peut maintenant compléter la preuve du théorème 1.2.

Démonstration du théorème 1.2 (b). On ne suppose vérifiées que les hypothèses I, II et III-(i). Soient $Y \in \mathscr{L}_{1}$ et $A^{Y^{\perp}}, F^{Y^{\perp}}$ les opérateurs du lemme 4.3, correspondant à $Z=Y^{\perp}$. Le calcul qui a mené à (5.8) montre que l'on a

$$
\left[H_{Y}, 1_{Y} \otimes A^{Y^{\perp}}\right]=2 \rho^{Y} \otimes \nabla_{Y^{\perp}}^{*} F^{Y^{\perp}} \text { sur } C_{0}^{\infty}(X) .
$$

Alors $H_{Y} \in C^{1}\left(A_{Y^{\perp}} ; \mathscr{H}^{1}(X), \mathscr{H}^{-1}(X)\right)$ si $A_{Y^{\perp}}:=1_{Y} \otimes A^{Y^{\perp}}$ et d'après le théorème 2.4, pour tout $u \in D\left(H_{Y}\right)$, fonction propre de $H_{Y}$ correspondant à $\lambda \in \sigma_{p}\left(H_{Y}\right)$, et tout $\varepsilon \in(0,1]$, on a

$$
\left.0=\left({ }_{[}^{[} H_{Y}, A_{Y^{\perp}}\right] u, u\right)=-2 i \int_{Y} \rho^{Y}(y)\left\{\int_{Y^{\perp}}|\eta|^{2} \hat{\psi}(\varepsilon \eta)|\hat{u}(y, \eta)|^{2} d \eta\right\} d y,
$$

où $\hat{u}(y,$.$) désigne la transformée de Fourier sur Y^{\perp}$ de la fonction $u(y,$.$) pour$ $y \in Y$ fixé. On a $\hat{\psi}(0)=1$ et alors pour $\varepsilon \searrow 0$ on obtient $u=0$. Q.E.D.

\section{§6. L'inégalité de Mlourre}

On aura besoin du résultat technique suivant (dont la démonstration est celle du lemme 2.8 .5 de [1]):

Lemme 6.1. Soit $H$ un opérateur auto-adjoint sur l'espace de Hilbert complexe et séparable $\mathscr{H}$. On désigne par $E_{I}(H)$ le projecteur spectral de $H$ 
associé à l'intervalle $I \subset \mathbb{R}$ et par $I_{r}(\lambda)$ l'intervalle $[\lambda-r, \lambda+r]$. Soit $A$ un autre

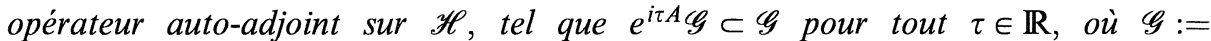
$D\left(|H|^{1 / 2}\right)$. On suppose de plus que $H \in C^{1}\left(A ; \mathscr{G}, \mathscr{G}^{*}\right)$ et qu'il existe $\lambda \in \mathbb{R}$, $r_{0}>0$ et $K \in \mathscr{K}\left(\mathscr{G}, \mathscr{G}^{*}\right)$ tels que l'on vérifie l'inégalité

$$
E_{I_{I_{0}}(\lambda)}(H) i[H, A] E_{I_{I_{0}}(\lambda)}(H) \geq a E_{I_{I_{0}}(\lambda)}(H)+K
$$

où $a \in \mathbb{R}(a \leq 0$ si $\lambda$ est une valeur propre de $H)$.

Alors, pour tout $\delta>0$, il existe $r>0$ tel que l'on ait

$$
E_{I_{(}(\lambda)}(H) i[H, A] E_{I_{1}(\lambda)}(H) \geq(a-\delta) E_{I_{(}(\lambda)}(H) .
$$

On remarque maintenant que la fonction $\rho$ a toutes les propriétés du potentiel $V$, donc on peut définir de la façon connue, pour tout $t \geq 0$, l'opérateur $H_{t}=H_{t}(\rho, V):=H(\rho, V+t \rho)$, qui sera auto-adjoint sur $L^{2}(X)$, $D\left(H_{t}\right)=D(H), \quad D\left(\left|H_{t}\right|^{1 / 2}\right)=D\left(|H|^{1 / 2}\right)=\mathscr{H}^{1}(X)$ et $H_{t}=H+t \rho$ sur $D(H)$. De plus, $H_{t} \in C^{1+\theta}(A)$.

De la même façon on définit pour tout $Y \in \mathscr{L}_{1}$, les opérateurs auto-adjoints $H_{Y, t}:=H_{Y}+t \rho_{Y}$ sur $L^{2}(X)$ et $H_{t}^{Y}:=H^{Y}+t \rho^{Y}$ sur $L^{2}(Y)$.

Nous allons d'abord étudier la dépendance en $t$ d'une fonction de l'opérateur $H_{t}$.

Lemme 6.2. Pour tout $f \in C_{0}^{\infty}(\mathbb{R})$, il existe une constante $C>0$ telle que l'on ait

$$
\begin{aligned}
& \left\|f\left(H_{t^{\prime}}\right)-f\left(H_{t^{\prime \prime}}\right)\right\|_{\mathscr{B}\left(\mathscr{H}^{-1}(X), \mathscr{H}^{1}(X)\right)} \\
& \quad \leq C\left(1+t^{\prime}\right)\left(1+t^{\prime \prime}\right)\left|t^{\prime}-t^{\prime \prime}\right|, \quad t^{\prime}, t^{\prime \prime} \in \mathbb{R}_{+} .
\end{aligned}
$$

Démonstration. On va utiliser la formule (3.5) pour $r \geq 3$. On remarque d'abord que pour $t^{\prime}, t^{\prime \prime} \in \mathbb{R}_{+}, \lambda \in \mathbb{R}, \tau \in \mathbb{R}^{*},|\tau| \leq 1$, on a dans $\mathscr{B}\left(\mathscr{H}^{-1}(X)\right.$, $\left.\mathscr{H}^{1}(X)\right)$ l'égalité suivante:

$$
\begin{aligned}
\left(H_{t^{\prime}}\right. & -\lambda-i \tau)^{-1}-\left(H_{t^{\prime \prime}}-\lambda-i \tau\right)^{-1} \\
& =\left(t-t^{\prime}\right)\left(H_{t^{\prime}}-\lambda-i \tau\right)^{-1} \rho^{Y}\left(H_{t^{\prime \prime}}-\lambda-i \tau\right)^{-1} .
\end{aligned}
$$

D'autre part, on vérifie facilement (voir aussi la démonstration du lemme 3.2) qu'il existe une constante $C_{0}$ telle que pour $t \in \mathbb{R}_{+}, \lambda, \tau \in \mathbb{R}, 0<|\tau| \leq 1$, on ait

$$
\left\|\left(H_{t}-\lambda-i \tau\right)^{-1}\right\|_{\mathscr{B}\left(L^{2}(X), \mathscr{H}^{1}(X)\right)} \leq C_{0}|\tau|^{-1}\left(1+t^{2}+\lambda^{2}\right)^{1 / 2} .
$$

On aura la même estimation pour la norme dans l'espace des opérateurs bornés de $\mathscr{H}^{-1}(X)$ dans $L^{2}(X)$.

Alors (6.3) résulte de (3.5), (6.4) et (6.5).

Q.E.D. 
On définit les seuils de $H_{t}$ par $\tau\left(H_{t}\right):=\bigcup_{Y \in \mathscr{L}_{1}} \sigma_{p}\left(H_{t}^{Y}\right)$. Pour $Y=\{0\}$, on a par convention $L^{2}(Y):=\mathbb{C}$ et $H_{t}^{Y}=V^{0}+t \rho^{0}$, donc $V^{0}+t \rho^{0} \in \tau\left(H_{t}\right)$.

On utilise les notations du lemme 6.1 pour les projecteurs spectraux et pour $a \in \mathbb{R}, t \geq 0$ on introduit la fonction $s_{a, t}: \mathbb{R} \rightarrow \mathbb{R}, s_{a, t}(\lambda)=a$ si $\lambda \notin \tau\left(H_{t}\right)$, $s_{a, t}(\lambda)=0$ si $\lambda \in \tau\left(H_{t}\right)$.

Proposition 6.3. Sous les hypothèses I-IV, on a:

(a) Pour tout $t \geq 0, \tau\left(H_{t}\right)$ est un ensemble dénombrable et fermé.

(b) Pour tout $t \geq 0$, les éléments de $\sigma_{p}\left(H_{t}\right) \backslash \tau\left(H_{t}\right)$ sont des valeurs propres de multiplicité finie, qui ne peuvent s'accumuler quaux points de $\tau\left(H_{t}\right)$. En particulier, $\tau\left(H_{t}\right) \cup \sigma_{p}\left(H_{t}\right)$ est un ensemble dénombrable et fermé.

(c) Soit $\lambda \in \mathbb{R}$. On peut choisir le nombre $\varepsilon>0$ qui intervient dans la définition de $A$ au $\S 4$ de façon que pour tout $T>0$ il existe $a>0$ tel que, pour tout $\delta>0$, il existe $r>0$ et tel que pour tout $t \in[0, T]$ il existe $K \in$ $\mathscr{K}\left(\mathscr{H}^{1}(X), \mathscr{H}^{-1}(X)\right)$ symétrique vérifiant l'inégalité

$$
E_{I_{I}(\lambda)}\left(H_{t}\right) i\left[H_{t}, A\right] E_{I_{1}(\lambda)}\left(H_{t}\right) \geq\left[s_{a, t}(\lambda)-\delta\right] E_{I_{I}(\lambda)}\left(H_{t}\right)+K .
$$

Démonstration. On prouve par induction sur $Y \in \mathscr{L}$ que les affirmations de la proposition 6.3 sont vraies pour tous les opérateurs $H_{t}^{Y}$.

(i) Pour $Y=\{0\}$ la proposition est évidemment vraie.

(ii) Avant de continuer, il faut préciser le choix de $\varepsilon>0$ pour $\lambda \in \mathbb{R}$ fixé.

Soient $m_{\gamma}:=\inf \sigma\left(H^{Y}\right), M:=\max _{Y \in \mathscr{L}_{1}}\left(\lambda+1-m_{Y}\right)\left\|\left(\rho^{Y}\right)^{-1}\right\|_{L^{\infty}(Y)}$ et $G_{Y, \varepsilon}: Y^{\perp}$ $\rightarrow \mathbb{R}, G_{Y, \varepsilon}(\eta):=2 \sum_{Z \in \mathscr{L}_{m}\left(Y^{\perp}\right)}\left|\pi_{Z} \eta\right|^{2} \hat{\psi}_{Z}\left(\varepsilon \pi_{Z} \eta\right), \eta \in Y^{\perp}$, où $\psi_{Z} \in C_{0}^{\infty}(Z)$ est la fonction qui a servi à la construction de $A^{Z}$ au début du $\S 4$. On a $G_{Y, \varepsilon}(\eta) \geq 0$ et $G_{Y, 0}(\eta)=2|\eta|^{2}$ pour tout $\eta \in Y^{\perp}$. Alors, si $M \leq 0$, le choix de $\varepsilon \in(0,1]$ est arbitraire, tandis que pour $M>0$, on choisit $\varepsilon \in(0,1]$ tel que $G_{Y, \varepsilon}(\eta) \geq|\eta|^{2}$ pour tout $\eta \in Y^{\perp}$ tel que $|\eta|^{2} \leq M$.

(iii) On suppose maintenant que pour un $Z \in \mathscr{L}$ fixé, la proposition est vraie pour tous les opérateurs $H_{t}^{Y}$ avec $t \geq 0$ et $Y \in \mathscr{L}(Z), Y \neq Z$. Il faut prouver la même proposition pour $H_{t}^{Z}, t \geq 0$. On pourra évidemment supposer $Z=X$.

(iv) Prouvons d'abord (a). $\tau\left(H_{t}\right)=\bigcup_{Y \in \mathscr{L}_{1}}\left[\tau\left(H_{t}^{Y}\right) \cup \sigma_{p}\left(H_{t}^{Y}\right)\right]$, donc d'après l'hypothèse d'induction, $\tau\left(H_{t}\right)$ est un ensemble dénombrable et fermé.

(v) Démontrons maintenant que $(\mathrm{a})$ et $(\mathrm{c})$ entraînent $(\mathrm{b})$. D'après (6.6) et la remarque 2.7, pour tout $t \geq 0$ et tout $\lambda \in \mathbb{R} \backslash \tau\left(H_{t}\right)$, il existe $r>0$ tel que $H_{t}$ ne peut avoir qu'un nombre fini de valeurs propres dans l'intervalle $I_{r}(\lambda)=$ $[\lambda-r, \lambda+r]$, chacune étant de multiplicité finie, ce qui implique (b).

(vi) Il reste à prouver que, si pour $\lambda \in \mathbb{R}, Y \in \mathscr{L}_{1}, T_{0}>0$, il existe $a_{Y}>0$, tel que pour tout $\delta^{\prime}>0$, il existe $r_{0}>0$, tel que pour tout $t \in\left[0, T_{0}\right]$, il existe $K_{Y} \in \mathscr{K}\left(\mathscr{H}^{1}(Y), \mathscr{H}^{-1}(Y)\right)$ symétrique vérifiant l'inégalité

$$
E_{I_{0}(\lambda)}\left(H_{t}^{Y}\right) i\left[H_{t}^{Y}, A^{Y}\right] E_{I_{(}(\lambda)}\left(H_{t}^{Y}\right) \geq\left[s_{a_{Y}, t}(\lambda)-\delta^{\prime}\right] E_{I_{r}(\lambda)}\left(H_{t}^{Y}\right)+K_{Y},
$$


où $s_{a_{Y}, t}$ est la fonction définie avant l'énoncé de la proposition 6.3, alors on a la relation (6.6).

(vii) On va prouver d'abord une inégalité de la forme (6.6) avec $K=0$ pour $H_{Y, t}, \quad Y \in \mathscr{L}_{1}, t \in[0, T], T>0$ arbitrairement fixé. $\mathrm{Si}$ on considère l'opérateur unitaire $\mathscr{F}_{Y}: L^{2}(X) \rightarrow \int_{Y^{\perp}}^{\oplus} L^{2}(Y) d \eta$, défini par la transformation de Fourier partielle

$$
\left(\mathscr{F}_{Y} u\right)(y, \eta):=(2 \pi)^{-(1 / 2) \operatorname{dim} Y^{\perp}} \int_{Y^{\perp}} e^{-i y^{\perp} \cdot \eta} u\left(y, y^{\perp}\right) d y^{\perp}, \quad u \in L^{2}(X),
$$

où $y \in Y, \eta \in Y^{\perp}$ et on tient compte de la relation (1.6), on voit que $H_{Y, t}$ est unitairement équivalent à l'intégrale directe suivante d'opérateurs auto-adjoints sur $L^{2}(Y)$ :

$$
\mathscr{F}_{Y} H_{Y, t_{Y}} \mathscr{F}_{Y}^{-1}=\int_{Y^{\perp}}^{\oplus} H_{t+|\eta|^{2}}^{Y} d \eta .
$$

D'autre part, si on désigne les projecteurs spectraux $E_{I,(\lambda)}$ par $E_{r}$ et on utilise l'identité (5.8), on aura l'égalité

$$
\mathscr{F}_{Y} E_{r}\left(H_{Y, t}\right) i\left(\left[H_{Y, t}, A\right] E_{r}\left(H_{Y, t}\right) \mathscr{F}_{Y}^{-1}=\int_{Y^{\perp}}^{\oplus} E_{r}\left(H_{t+|\eta|^{2}}^{Y}\right) B_{t}^{Y}(\eta) E_{r}\left(H_{t+|\eta|^{2}}^{Y}\right) d \eta\right.
$$

où

$$
B_{t}^{Y}(\eta)=i\left[H_{t+|\eta|^{2}}^{Y}, A^{Y}\right]+G_{Y, \varepsilon}(\eta) \rho^{Y} \in \mathscr{B}\left(\mathscr{H}^{1}(Y), \mathscr{H}^{-1}(Y)\right)
$$

est symétrique. Pour minorer l'intégrand de (6.9) pour $r<1$, on voit que le seul cas intéressant est celui où, pour $M$ défini au (ii), on a $M>0$ et $|\eta|^{2} \leq M$.

En effet, si $M \leq 0$, on a $\lambda+1 \leq m_{Y}=\inf \sigma\left(H^{Y}\right) \leq \inf \sigma\left(H_{t+|\eta|^{2}}^{Y}\right)$, donc $E_{r}\left(H_{t+|\eta|^{2}}^{Y}\right)=0$. Si $M>0$ et $|\eta|^{2} \geq M$, on a $H_{t+|\eta|^{2}}^{Y} \geq m_{Y}+M \rho^{Y} \geq \lambda+1$ donc $E_{r}\left(H_{t+|\eta|^{2}}^{Y}\right)=0$.

Pour étudier le cas $|\eta|^{2} \leq M$, on voit par l'hypothèse d'induction (6.7) (où l'on fixe $T_{0}:=T+M$ ) et le lemme 6.1 , que pour tout $\delta^{\prime}>0$, tout $\eta \in Y^{\perp}$ avec $|\eta|^{2} \leq M$ et tout $t \in[0, T]$, il existe $r_{0}=r_{0}\left(\delta^{\prime}, \eta, t\right)>0$ tel que l'on vérifie l'inégalité

$$
E_{r_{0}}\left(H_{t+|\eta|^{2}}^{Y}\right) i\left[H_{t+|\eta|^{2}}^{Y}, A^{Y}\right] E_{r_{0}}\left(H_{t+|\eta|^{2}}^{Y}\right) \geq\left[s_{a_{Y}, t+|\eta|^{2}}(\lambda)-2 \delta^{\prime}\right] E_{r_{0}}\left(H_{t+|\eta|^{2}}^{Y}\right) .
$$

En particulier, si on choisit ci-dessus $\eta=0$ et on considère pour tout $t \in[0, T]$ une fonction réelle $f=f_{t} \in C_{0}^{\infty}(\mathbb{R})$, supp $f \subset I_{r_{0}}(\lambda), f=1$ sur $I_{r_{0} / 2}(\lambda)$, on $\mathrm{a}$

$$
f\left(H_{t}^{Y}\right) i\left[H_{t}^{Y}, A^{Y}\right] f\left(H_{t}^{Y}\right) \geq\left[S_{a_{Y}, t}(\lambda)-2 \delta^{\prime}\right] f\left(H_{t}^{Y}\right)^{2} .
$$

Avant de poursuivre, il faut étudier la dépendance en $t$ et $\eta$ de certains opérateurs intervenant dans les relations précédentes. D'après le lemme 6.2, 
pour tout $f \in C_{0}^{\infty}(\mathbb{R})$ il existe une constante $C>0$ telle que l'on ait:

$$
\left\|f\left(H_{t^{\prime}+\left|\eta^{\prime}\right|^{2}}^{Y}\right)-f\left(H_{t^{\prime \prime}+\left|\eta^{\prime \prime}\right|^{2}}^{Y}\right)\right\|_{\mathscr{B}\left(\mathscr{H}^{-1}(Y), \mathscr{H}^{1}(Y)\right)} \leq C\left(\left|t^{\prime}-t^{\prime \prime}\right|+\left.|| \eta^{\prime}\right|^{2}-\left|\eta^{\prime \prime}\right|^{2} \mid\right)
$$

pour tous $t^{\prime}, t^{\prime \prime} \in[0, T], \eta^{\prime}, \eta^{\prime \prime} \in Y^{\perp},\left|\eta^{\prime}\right|^{2} \leq M,\left|\eta^{\prime \prime}\right|^{2} \leq M$.

Il est évident aussi qu'il existe une autre constante $C_{1} \geq 0$ telle que l'on ait pour les mêmes valeurs de $t^{\prime}, t^{\prime \prime}, \eta^{\prime}, \eta^{\prime \prime}$ :

$$
\begin{gathered}
\left\|\left[H_{t^{\prime}+\left.\eta^{\prime}\right|^{2}}^{Y}, A\right]-\left[H_{t^{\prime \prime}+\mid \eta^{\prime \prime} 1^{2}}^{Y}, A\right]\right\|_{\mathscr{B}\left(\mathscr{H}^{-1}(Y), \mathscr{H}^{1}(Y)\right)} \\
\leq C_{1}\left(\left|t^{\prime}-t^{\prime \prime}\right|+\left.|| \eta^{\prime}\right|^{2}-\left|\eta^{\prime \prime}\right|^{2} \mid\right) \\
\left\|B_{t^{\prime}}^{Y}\left(\eta^{\prime}\right)-B_{t^{\prime \prime}}^{Y}\left(\eta^{\prime \prime}\right)\right\|_{\mathscr{B}\left(\mathscr{H}^{-1}(Y), \mathscr{H}^{1}(Y)\right)} \leq C_{1}\left(\left|t^{\prime}-t^{\prime \prime}\right|+\left|\eta^{\prime}-\eta^{\prime \prime}\right|\right)
\end{gathered}
$$

et finalement, pour tous $t \in[0, T]$ et $\eta \in Y^{\perp},|\eta|^{2} \leq M$ :

$$
\left\|B_{t}^{Y}(\eta)-B_{t}^{Y}(0)\right\|_{\mathscr{B}\left(\mathscr{H}^{1}(Y), \mathscr{H}^{-1}(Y)\right)} \leq C_{1}|\eta|^{2} .
$$

En utilisant (6.12), (6.13) et (6.16), on voit que pour tout $t \in[0, T]$, il existe une constante positive $c=c(t)$ telle que pour tout $\eta \in Y^{\perp},|\eta|^{2} \leq c \delta^{\prime}$, il existe $R_{1}=$ $R_{1}(t, \eta) \in \mathscr{B}\left(\mathscr{H}^{-1}(Y), \mathscr{H}^{1}(Y)\right)$ tel que $\left\|R_{1}\right\|_{\mathscr{B}\left(\mathscr{H}^{-1}(Y), \mathscr{H}^{1}(Y)\right)} \leq \delta^{\prime}$ et

$$
f\left(H_{t+|\eta|^{2}}^{Y}\right) B_{t}(\eta) f\left(H_{t+|\eta|^{2}}^{Y}\right) \geq\left[s_{a_{Y}, t}(\lambda)-2 \delta^{\prime}\right] f\left(H_{t+|\eta|^{2}}^{Y}\right)^{2}-R_{1} .
$$

D'après (6.17), (6.13) et (6.15), pour tout $t \in[0, T]$, il existe une constante positive $\sigma=\sigma(t)$ telle que pour tout $t^{\prime} \in[0, T],\left|t^{\prime}-t\right| \leq \sigma$ et tout $\eta \in Y^{\perp}, \quad|\eta|^{2} \leq c \delta^{\prime}, \quad$ il existe $R_{2}=R_{2}\left(t, t^{\prime}, \eta\right) \in \mathscr{B}\left(\mathscr{H}^{-1}(Y), \mathscr{H}^{1}(Y)\right)$, tel que $\left\|R_{2}\right\|_{\mathscr{B}\left(\mathscr{H}^{-1}(Y), \mathscr{H}^{1}(Y)\right)} \leq \delta^{\prime}$ et

$$
f\left(H_{t^{\prime}+|\eta|^{2}}^{Y}\right) B_{t^{\prime}}(\eta) f\left(H_{t^{\prime}+|\eta|^{2}}^{Y}\right) \geq\left[s_{a_{Y}, t}(\lambda)-2 \delta^{\prime}\right] f\left(H_{t^{\prime}+|\eta|^{2}}^{Y}\right)^{2}-R_{1}-R_{2} .
$$

Si on considère un recouvrement fini de $[0, T]$, on trouve donc qu'il existe une constante $c_{1}>0$ telle que pour tout $\delta^{\prime}>0$, il existe $r_{1}=r_{1}\left(\delta^{\prime}\right)>0$ tel que pour tous $t \in[0, T]$ et $\eta \in Y^{\perp},|\eta|^{2} \leq c_{1} \delta^{\prime}$, on ait la relation:

$$
E_{r_{1}}\left(H_{t+|\eta|^{2}}^{Y}\right) B_{t}(\eta) E_{r_{1}}\left(H_{t+|\eta|^{2}}^{Y}\right) \geq\left[s_{a_{Y}, t}(\lambda)-4 \delta^{\prime}\right] E_{r_{1}}\left(H_{t+|\eta|^{2}}^{Y}\right) .
$$

On remarque maintenant en utilisant (6.11), (6.10) et l'inégalité $G_{Y, \varepsilon}(\eta) \geq$ $|\eta|^{2}$ si $\eta \in Y^{\perp},|\eta|^{2} \leq M$ qu'il existe une constante $\alpha>0$, telle que pour tout $\delta^{\prime \prime}>0$, tout $\eta \in Y,|\eta|^{2} \leq M$ et tout $t \in[0, T]$, il existe $r_{0}=r_{0}\left(\delta^{\prime \prime}, \eta, t\right)>0$, tel que l'on vérifie l'inégalité:

$$
E_{r_{0}}\left(H_{t+|\eta|^{2}}^{Y}\right) B_{t}(\eta) E_{r_{0}}\left(H_{t+|\eta|^{2}}^{Y}\right) \geq\left(\alpha|\eta|^{2}-\delta^{\prime \prime}\right) E_{r_{0}}\left(H_{t+|\eta|^{2}}^{Y}\right)
$$

Si $\lambda \in \tau\left(H_{t}\right)$ on choisit dans (6.19) $\delta^{\prime}=\frac{1}{4} \delta, \delta>0$ et dans $(6.20) \delta^{\prime \prime}=\delta$. 
Si $\lambda \notin \tau\left(H_{t}\right)$, on a $s_{a_{Y}, t}(\lambda)=a_{Y}$ et on choisit dans (6.19), $\delta^{\prime}=\frac{a_{Y}}{8}$ et dans (6.20), $\delta^{\prime \prime}=\alpha c_{1} a_{Y} / 16$. Il en résulte qu'il existe une constante $a>0$, telle que pour tout $\delta>0$, tout $t \in[0, T]$ et tout $\eta \in Y^{\perp},|\eta|^{2} \leq M$, il existe $r_{2}=$ $r_{2}(\delta, \eta, t)>0$ tel que l'on ait

$$
E_{r_{2}}\left(H_{t+|\eta|^{2}}^{Y}\right) B_{t}(\eta) E_{r_{2}}\left(H_{t+|\eta|^{2}}^{Y}\right) \geq\left[s_{a, t}(\lambda)-\delta\right] E_{r_{2}}\left(H_{t+|\eta|^{2}}^{Y}\right) .
$$

On voit facilement que l'on peut choisir $r_{2}$ indépendant de $t \in[0, T]$ et $\eta \in Y^{\perp},|\eta|^{2} \leq M$. Il suffit de suivre le raisonnement qui a mené de (6.11) à (6.19): pour $t \in[0, T]$ et $\eta \in Y^{\perp},|\eta|^{2} \leq M$ fixés, on considère une fonction réelle $f=f_{t, \eta} \in C_{0}^{\infty}(\mathbb{R}), \operatorname{supp} f \subset I_{r_{2}}(\lambda), f=1$ sur $I_{r_{2} / 2}(\lambda)$, on multiplie (6.21) à gauche et à droite avec $f\left(H_{t+|\eta|^{2}}^{Y}\right)$, on utilise (6.13) et (6.15), ainsi qu'un recouvrement fini de $\left\{(t, \eta) \in[0, T] \times Y^{\perp} ;|\eta|^{2} \leq M\right\}$.

En utilisant (6.9) et (6.21), on déduit l'existence d'une constante $a>0$ telle que pour tout $\delta>0$, il existe $r>0$ tel que l'on vérifie l'inégalité

$$
E_{r}\left(H_{Y, t}\right) i\left[H_{Y, t}, A\right] E_{r}\left(H_{Y, t}\right) \geq\left[s_{a, t}(\lambda)-\delta\right] E_{r}\left(H_{Y, t}\right)
$$

pour tous $Y \in \mathscr{L}_{1}$ et $t \in[0, T]$.

(viii) Pour démontrer (6.6), on considère $f \in C_{0}^{\infty}(\mathbb{R})$ réelle, telle que $\operatorname{supp} f$ $\subset I_{r}(\lambda), f=1$ sur $I_{r / 2}(\lambda)$. La relation (6.22) entraîne alors l'inégalité

$$
f\left(H_{Y, t}\right) i\left[H_{Y, t}, A\right] f\left(H_{Y, t}\right) \geq\left(s_{a, t}(\lambda)-\delta\right) f\left(H_{Y, t}\right)^{2} .
$$

On utilise maintenant la relation (5.5) pour $H_{t}$, avec $S=S(t) \in \mathscr{B}\left(\mathscr{H}^{1}(X)\right.$, $\left.\mathscr{H}^{-1}(X)\right)$ et \langle\rangle$^{\theta} S \in \mathscr{B}\left(\mathscr{H}^{1}(X), \mathscr{H}^{-1}(X)\right)$. Alors $f\left(H_{Y, t}\right) S f\left(H_{Y, t}\right)=\langle.\rangle^{-\theta}$. $f\left(H_{Y, t}\right)\langle.\rangle^{\theta} S f\left(H_{Y, t}\right)+\left[f\left(H_{Y, t}\right),\langle.\rangle^{-\theta}\right]\langle.\rangle^{\theta} S f\left(H_{Y, t}\right)$, opérateur qui, d'après le lemme 3.2, est compact sur $L^{2}(Y)$. Si on désigne par $\sim$ l'égalité modulo un opérateur de $\mathscr{K}\left(L^{2}(Y)\right)$ et on utilise les lemmes 3.2 et 3.7 , ainsi que les relations (3.10), (5.5) et (6.23), on aura

$$
\begin{aligned}
f\left(H_{t}\right) i\left[H_{t}, A\right] f\left(H_{t}\right) & \sim \sum_{Y \in \mathscr{L}_{1}} \chi_{Y} f\left(H_{t}\right) i\left[H_{Y, t}, A\right] f\left(H_{t}\right) \chi_{Y} \\
& \sim \sum_{Y \in \mathscr{L}_{1}} \chi_{Y} f\left(H_{Y, t}\right) i\left[H_{Y, t}, A\right] f\left(H_{Y, t}\right) \chi_{Y} \\
& \geq\left[s_{a, t}(\lambda)-\delta\right] \sum_{Y \in \mathscr{L}_{1}} \chi_{Y} f\left(H_{Y, t}\right)^{2} \chi_{Y} \sim\left[s_{a, t}(\lambda)-\delta\right] f\left(H_{t}\right)^{2} .
\end{aligned}
$$

On en déduit qu'il existe $a>0$ tel que pour tout $\delta>0$, il existe $r>0$, tel que pour tout $t \in[0, T]$, il existe $K \in \mathscr{K}\left(L^{2}(X)\right)$ auto-adjoint tel que l'on vérifie (6.6) pour $I_{r / 2}(\lambda)$.

Q.E.D.

Démonstration du théorème 1.3 .

Les affirmations (a) et (b) résultent de la proposition 6.3 pour $t=0$. 
L'affirmation (c) est une conséquence du théorème 2.8, car d'après la proposition 5.4, $H \in C^{1+\theta}\left(A ; \mathscr{H}^{1}(X), \mathscr{H}^{-1}(X)\right)$ et la proposition 6.3 entraîne le fait que pour tout $\lambda \in \mathbb{R} \backslash \tau(H)$ il existe $\varepsilon>0$ et $\delta>0$ tels que l'intervalle $(\lambda-\delta, \lambda+\delta)$ soit inclus dans $\mu^{A}(H)$.

\section{Démonstration du théorème 1.4.}

(a) Pour appliquer le théorème 2.8 on tient compte du fait suivant: si $\quad \mathscr{H}:=L^{2}(X), \quad \mathscr{G}:=\mathscr{H}^{1}(X), \quad \mathscr{G}^{*}=\mathscr{H}^{-1}(X), \quad$ alors $\quad \mathscr{H}_{1}^{-1}(X) \subset D\left(A ; \mathscr{G}^{*}\right)$ et donc $\left(\mathscr{H}^{-1}(X), \mathscr{H}_{1}^{-1}(X)\right)_{(1 / 2), 1} \subset \mathscr{E}:=\left(\mathscr{G}^{*}, D\left(A ; \mathscr{G}^{*}\right)\right)_{(1 / 2), 1}$. D'après [4], $\left(\mathscr{H}^{-1}(X), \mathscr{H}_{1}^{-1}(X)\right)_{(1 / 2), 1}=\mathscr{H}_{(1 / 2), 1}^{-1}(X)$ et l'espace adjoint sera $\left.\mathscr{H}_{-(1 / 2), \infty}^{1}(X)\right)$, donc $\mathscr{B}\left(\mathscr{E}, \mathscr{E}^{*}\right) \subset \mathscr{B}\left(\mathscr{H}_{(1 / 2), 1}^{-1}(X), \mathscr{H}_{-(1 / 2), \infty}^{1}(X)\right)$.

(b) On sait que pour tous $\gamma>\frac{1}{2}$ et $\varepsilon>0$ tels que $\frac{1}{2}+\varepsilon<\gamma$, les injections canoniques $\mathscr{H}_{\gamma}^{0}(X) \subset \mathscr{H}_{(1 / 2)+\varepsilon}^{-1}(X) \subset \mathscr{H}_{(1 / 2), 1}^{-1}(X)$ et $\mathscr{H}_{-(1 / 2), \infty}^{1}(X) \subset$ $\mathscr{H}_{-(1 / 2)-\varepsilon}^{1}(X) \subset \mathscr{H}_{-\gamma}^{0}(X)$ sont bornées, la première et la dernière étant même compactes. On déduit du (a) que les applications holomorphes

$$
\mathbb{C}^{ \pm} \ni z \mapsto(H-z)^{-1} \in \mathscr{B}\left(\mathscr{H}_{\gamma}^{0}, \mathscr{H}_{-\gamma}^{0}\right), \quad \gamma>\frac{1}{2},
$$

admettent des extensions continues pour la topologie de la norme à $\mathbb{C}^{ \pm} \cup$ $\left(\mathbb{R} \backslash\left(\tau(H) \cup \sigma_{p}(H)\right)\right.$.

Il ne reste qu'à remarquer l'identité

$$
\begin{gathered}
(H-\lambda)^{-1}=(H-i)^{-1}+(\lambda-i)(H-i)^{-2}+(\lambda-i)^{2}(H-i)^{-1}(H-\lambda)^{-1}(H-i)^{-1}, \\
\lambda \in \mathbb{C}^{ \pm},
\end{gathered}
$$

où $(H-i)^{-1} \in \mathscr{B}\left(\mathscr{H}^{-1}(X), \mathscr{H}^{1}(X)\right)$ et le fait que d'après le lemme 3.1, $(H-i)^{-1} \in \mathscr{B}\left(\mathscr{H}_{t}^{-1}(X), \mathscr{H}_{t}^{1}(X)\right)$ pour tout $t \in[-1,1]$.

Q.E.D.

\section{Références}

[1] Amrein, W., Boutet de Monvel, A. and Georgescu, V., Notes on the $N$-body problem, II (1988), II (1991), Preprint Université de Genève.

[2] —,$C_{0}$-groups, commutator methods and spectral theory of $N$-body hamiltonians, Progr. Math., Birkhäuser, 1996.

[3] Bergh, J. and Löfström, J., Interpolation spaces: an introduction, Springer Verlag, 1976.

[4] Boutet de Monvel, A. and Georgescu, V., Spectral and scattering theory by the conjugate operator method, Algebra $i$ Analiz, 4:3 (1992), 73-116.

[5] - Some developments and applications of the abstract Mourre theory, Astérisque, $210: 2$ (1992), 27-48.

[6] Boutet de Monvel, A. and Manda, D., Spectral and scattering theory for wave propagation in perturbed stratified media, J. Math. Anal. Appl., 191 (1995), 137-167.

[7] Boutet de Monvel, A., Manda, D. and Purice, R., The commutator method for formrelatively compact perturbations, Lett. Math. Phys., 22 (1991), 211-223. 
[8] Croc. E. and Dermenjian, Y., A perturbative method for the spectral analysis of an acoustic multistratified strip, Math. Meth. Appl. Sci., 21 (1998), 1681-1704.

[9] Cycon, H. L., Froese, R. G., Kirsch, W. and Simon, B., Schrödinger operators, Springer Verlag, 1987.

[10] De Bièvre, S. and Pravica, D. W., Spectral analysis for optical fibres and stratified fluids, J. Funct. Anal., 98 (1991), 404-436.

[11] Dermenjian, Y., Durand, M. and Iftimie, V., Spectral analysis of an acoustic multistratified perturbed cylinder, Comm. PDE, 23:1-2 (1998), 141-169.

[12] Froese, R. G. and Herbst, I., A new proof of the Mourre estimate, Duke Math. J., 49 (1982), 1075-1085.

[13] Iftimie, V., Opérateurs différentiels magnétiques: stabilité des trous dans le spectre, invariance du spectre essentiel et applications, Comm. PDE, 18:3-4 (1993), 651-686.

[14] Mourre, E., Absence of singular continuous spectrum for certain self-adjoint operators, Comm. Math. Phys., 78 (1981), 391-408.

[15] Perry, P., Sigal, I. M. and Simon, B., Spectral analysis of $N$-body Schrödinger operators, Ann. Math., 114 (1981), 519-567. 
